\title{
Extreme binary forms
}

by

Andrzej Białynicki-Birula and Andrzej Schinzel (Warszawa)

1. Introduction. Let $K$ be an algebraically closed field of characteristic 0 or $>d$, where $d$ is a positive integer. Let $f$ be a binary form in $K[x, y]$ of degree $d$. By a presentation of $f$ we mean a decomposition

$$
f=l_{1}^{d}+\cdots+l_{u}^{d}
$$

of $f$ into a sum of powers of linear forms $l_{1}, \ldots, l_{u} \in K[x, y]$. Let $r=r(f)$ be the minimal length of a presentation of $f$. Then $f$ is said to be extreme if for every linear form $l \in K[x, y]$, the sum $f+l^{d}$ admits a presentation of length $r(f)$. In other words, a form is extreme if and only if it is maximal with respect to the partial order denoted by $\prec$ and defined by: a form $f$ is greater than every form obtained as a sum of some, but not all, summands appearing in a presentation of $f$ of minimal length.

A form is said to be exotic if the minimal length of presentation of $f$ is greater than the minimal length of presentation of a generic form of the same degree. Hence exotic forms are defined as those that have the minimal length of presentation greater than the length predicted by an answer to the Waring Problem. In the case of binary forms, the minimal length $r_{0}$ of presentation of a general binary form of degree $d$ is

$$
(d+1) / 2 \text { in case } d \text { is odd and }(d+2) / 2 \text { in case } d \text { is even. }
$$

Hence a binary form of degree $d$ is exotic if its minimal length of presentation is greater than $(d+2) / 2$. A form of degree $d$ is said to be regular if its minimal length of presentation is equal to the length of presentation of a general form of degree $d$. A form of degree $d$ is said to be plain if its minimal length of presentation is less than the length of presentation of a general form of degree $d$.

When we consider presentations of forms as sums of forms, then usually we are not able to identify projectively equal forms and simultaneously 
identify their projectively equal summands. Hence, in the following, we are going to treat forms as elements of the vector space $K[x, y]$, rather than of its projectivization. The properties of forms which we are going to study are invariant under invertible linear substitutions, i.e. under the natural action of $G L(2)$ on $K[x, y]$. Moreover, the studied notions have some interpretation, easy to find but not helpful in our considerations, in the geometric theory of secant varieties obtained as the images of the projective line under Veronese embedding into a projective space.

Let $K[X, Y]$ be the dual ring of differential operators, where

$$
X=\frac{\partial}{\partial x}, \quad Y=\frac{\partial}{\partial y} .
$$

Let $K[X, Y]_{t}$ denote the space of homogeneous operators of degree $t$, $t=0,1, \ldots$ For $f \in K[x, y]$, let $A(f) \subset K[X, Y]$ be the annihilating ideal of $f$ and $A_{t}(f)$ its homogeneous part of degree $t$, i.e.

$$
A_{t}(f)=K[X, Y]_{t} \cap A(f) .
$$

We shall say that a form $D \in K[X, Y]$ is squareful if, for a linear form $L$, whenever $L \mid D$, then $L^{2} \mid D$.

One of the main aims of the paper is to characterize properties of forms by means of properties of annihilating differential operators. Let $j=\lfloor(d+2) / 2\rfloor$. We shall see that a form $f \in K[x, y]_{d}$ is

- regular if and only if there exist projectively distinct linear differential operators $D_{1}, \ldots, D_{j}$ such that $D_{1} \ldots D_{j}(f)=0$ (Theorem 1),

- plain if and only if there exist projectively distinct linear differential operators $D_{1}, \ldots, D_{u}, u<j$, such that $D_{1} \ldots D_{u}(f)=0$ (Theorem 1),

- exotic if and only if no squarefree differential operator of degree $\leq j$ annihilates $f$ (Theorem 1),

- extreme and exotic if and only if it is annihilated by a squareful linear differential operator $D$ of degree $u<j$ (Theorem $1^{\prime}$ ),

- exotic and regular if and only if (this is the most difficult case) the conditions given in Theorem 5 are satisfied (then $d$ has to be even).

Since there are no extreme plain forms (Lemma 3.4), all cases are covered above.

It follows from these results that, for example, all forms $f=l^{m} g \in$ $K[x, y]_{d}$, where $l$ is a linear form, $g \in K[x, y]_{d-m}$ is relatively prime to $l$ and $2 m>d+1$, are extreme. Moreover, all extreme forms with minimal length of presentation equal to $d$ are equal to $l_{1}^{d-1} l_{2}$, where $l_{1}, l_{2}$ are projectively different linear forms (Corollary 3). This means that they constitute one $G L(2)$-orbit. The set of extreme forms with minimal length of presentation equal to $d-1, d \geq 6$, is composed of two orbits. For exotic forms these are the only cases where the exotic extreme forms with given minimal length of 
presentation comprise a finite number of $G L(2)$-orbits. However, the extreme regular forms (they exist only if $d=2 m$ ) comprise a finite number of orbits (Corollary 7). The proof of this fact is the most difficult part of the paper.

In the remaining part of the paper we study, for a binary form $f$, all presentations of $f$ as a sum of powers $l_{1}^{d}+\cdots+l_{u}^{d}$ of linear forms of minimal length $u$. In particular, we would like to know all partial summands $g=$ $l_{1}^{d}+\cdots+l_{t}^{d}, t<u$, that appear in such presentations. This leads to studying the $\prec$ relation. We start with the following simple observation. Plain forms and regular forms in case $d+1$ is even and only those forms have a unique presentation of minimal length. It follows that if, for a form $f=l_{1}^{d}+\cdots+l_{u}^{d}$, we have already fixed some part $g=l_{1}^{d}+\cdots+l_{t}^{d}, t<u, u-t<(d+1) / 2$, of its minimal decomposition, then the remaining part of the decomposition is already uniquely determined. If $f, g$ are as above and $g$ is regular or exotic, then the conditions are satisfied, hence, then, $f-g$ has a unique minimal presentation. Next we prove that, for $f$ a regular form of even degree or an exotic form, and only for such forms, there exist only finitely many extreme forms $h$ such that $f \prec h$, hence there are only finitely many forms greater than $f$ (Proposition 3 ).

REMARK. The map $r$ which assigns to a form $f$ of degree $d$ its minimal length of presentation $r(f)$ satisfies the triangle inequality $r(f+g) \leq r(f)+$ $r(g)$ and defines a metric $\rho$ (by $\rho(f, g)=r(f-g)$ ) on $K[x, y]_{d}$. Then $f \prec g$ if and only if $r(f)+r(g-f)=r(g)$. A form $h$ is extreme if and only if $r$ admits at $h$ its local maximum, more exactly, $r(h)$ is maximal among the values of $r$ taken in the closed ball of radius 1. In other words, the distance from an extreme form to 0 is locally maximal.

For every form $f$ we can consider the set of all linear forms $l \in K[x, y]_{1}$ such that $l^{d} \prec f$, i.e. $l^{d}$ appears in some minimal presentation of $f$. This set is finite for any plain form and any regular form in case $d+1$ is even, and infinite with non-empty complement in $K[x, y]_{1}$ composed of a finite number of lines in the remaining cases (Proposition 1). Studying these cases leads finally to the result (Corollary 5) that all forms can be divided into two disjoint classes: those that dominate only finitely many other forms and those that are dominated only by finitely many forms.

There are still some open problems connected with the results of the paper.

Problem. What is the number $n(m)$ of linear equivalence classes of regular extreme forms of degree $2 m$ ?

\section{Statements of the results}

THEOREM 1. Let $f$ be a binary form of degree $d>1$ with minimal length of presentation $r(f)=r$. Then $f$ is extreme if and only if $f$ is regular or 
exotic and

- whenever $f$ is exotic, then every element of $A_{d+2-r}(f)$ is squareful,

- whenever $f$ is regular, then $d$ is even and every element of $A_{d+2-r}(f)=$ $A_{(d+2) / 2}(f)$ is either squarefree or squareful.

We shall see that in the case where $f$ is exotic, $\operatorname{dim} A_{d+2-r}(f)=1$. Thus every element of $A_{d+2-r}(f)$ is squareful if and only if $A_{d+2-r}(f)$ contains a non-zero squareful form.

In order to formulate further assertions we need to quote some auxiliary results.

Lemma 2.1. Let $l_{1}, \ldots, l_{u}$ be projectively distinct non-zero linear forms and let $L_{1}, \ldots, L_{u}$ be non-zero linear operators such that $L_{i} l_{i}=0$ for $i=$ $1, \ldots, u$. Let $e_{1}+\cdots+e_{u}+u \leq d$. A binary form $f$ of degree $d$ admits a decomposition

$$
f=g_{1} l_{1}^{d-e_{1}}+\cdots+g_{u} l_{u}^{d-e_{u}},
$$

where, for $i=1, \ldots, u, g_{i} \in K[x, y]$ is a form of degree $e_{i}$ or 0 , if and only if

$$
L_{1}^{e_{1}+1} \ldots L_{u}^{e_{u}+1} f=0 .
$$

In particular, $f$ admits a presentation

$$
f=a_{1} l_{1}^{d}+\cdots+a_{u} l_{u}^{d},
$$

where $a_{1}, \ldots, a_{u} \in K$, if and only if

$$
L_{1} \ldots L_{u} f=0 .
$$

Proof. See [2, Lemma 1.31].

Lemma 2.2. Let $f$ be a binary form of degree $d$.

(i) The sequence $\left(\operatorname{dim} K[X, Y]_{u} / A_{u}(f)\right)_{u=0}^{d}$ has the following shape:

$$
(1,2, \ldots, s-1, s, \ldots, s, s-1, \ldots, 2,1),
$$

where $s=s(f):=\max _{0 \leq u \leq d} \operatorname{dim}\left(K[X, Y]_{u} / A_{u}(f)\right) \leq(d+2) / 2$.

(ii) The ideal $A(f)$ is generated by two relatively prime homogeneous forms $\mathcal{D}_{f}, \mathcal{E}_{f}$ of degree $s$ and $d-s+2$, respectively. For $s \leq(d+1) / 2$ the form $\mathcal{D}_{f}$ is uniquely determined up to projective equality and for $u=s, \ldots, d-s+1$ we have

$$
A_{u}(f)=\mathcal{D}_{f} K[X, Y]_{u-s} .
$$

Proof. For the proof of (i), see [2, Theorems 1.43 and 1.44(i)]. For the proof of (ii), see [2, Theorem 1.44(ii) and (iii) and Claim on p. 31].

Lemma 2.3. Let $F_{1}, \ldots, F_{u}$ be binary forms of the same degree such that the set of all forms $f=a_{1} F_{1}+\cdots+a_{u} F_{u}, a_{1}, \ldots, a_{u} \in K$, which are not squarefree is Zariski dense in the linear span of $F_{1}, \ldots, F_{u}$. Then there exists a linear form $L \in K[X, Y]$ such that $L^{2}$ divides all $F_{1}, \ldots, F_{u}$. 
Proof. See the proof of Lemma 1.1 in [3].

With the notation established in Lemma 2.2, we can formulate Theorem 1 in a slightly weaker, but more transparent form:

THEOREM 1'. A binary exotic form $f$ is extreme if and only if $\mathcal{D}_{f}$ is squareful.

COROLlary 1. If a binary form $f$ of degree $d$ has a linear factor of multiplicity $m$, where $(d+1) / 2 \leq m<d$, then $f$ is extreme.

COROLlary 2. If a binary form $f$ of degree $d \geq 5$ is extreme and $r(f) \geq$ $d-1$, then $f$ has a linear factor of multiplicity $r(f)-1$. For $d \geq 6$ there exists a squarefree extreme form with $r(f)=d-2$.

The special case of Corollary 2 for $r(f)=d$ is due to J. Kleppe [3, p. 11].

In view of Corollary 2 it is doubtful that extreme forms have a simple characterization in terms of factorization of $f$ instead of that of $\mathcal{D}_{f}$.

Then we show the following characterization of exotic extreme forms:

TheOrem 2. An exotic form $f$ is extreme if and only if it is of the shape

$$
f=g_{1} m_{1}^{d-e_{1}}+\cdots+g_{u} m_{u}^{d-e_{u}},
$$

where $m_{1}, \ldots, m_{u}$ are pairwise projectively distinct linear forms, $g_{1}, \ldots, g_{u}$ are forms of positive degree $e_{1}, \ldots, e_{u}$, respectively, $m_{i} \nmid g_{i}$ and

$$
e_{1}+\cdots+e_{u}+u<\frac{d+2}{2} .
$$

Moreover, for $f$ as above, if $M_{1}, \ldots, M_{u}$ are non-zero linear operators such that $M_{i} m_{i}=0$ for $i=1, \ldots, u$, then

$$
\mathcal{D}_{f}=M_{1}^{e_{1}+1} \ldots M_{u}^{e_{u}+1} .
$$

The following corollary follows immediately from the above Theorem 2 .

COROLlary 3. Up to a linear transformation we have only the following extreme forms $f_{0}$ with the given lengths of minimal presentations:

1. $r\left(f_{0}\right)=d, d \geq 2$, only $f_{0}=x y^{d-1}$.

2. $r\left(f_{0}\right)=d-1, d \geq 4$, only $f_{0}=x(x+y) y^{d-2}$ and $f_{0}=x^{2} y^{d-2}$. For $d \leq 7$ these are all exotic extreme forms and for $d<6$ these are all extreme forms.

3. For $r\left(f_{0}\right)=d-2, d \geq 7$, we have two infinite families of extreme exotic forms which contain all extreme forms: $f_{0}=x(x+y)(x+a y) y^{d-3}$, $f_{0}=x y^{d-1}+a x^{d-1} y$, where $a \in K \backslash\{0\}$.

4. For $r\left(f_{0}\right)=d-3, d \geq 9$, we have two infinite families of extreme exotic forms which contain all extreme forms: $f_{0}=x(x+y)(x+a y)$ $\cdot(x+b y) y^{d-4}$ and $f_{0}=(x+a y) y^{d-1}+e(b x+y)(c x+y) x^{d-2}$, where $a, b, c \in K, e \in K \backslash\{0\}$. 
Moreover, for $d=2 m$ we have a regular extreme form $f_{0}=x^{m} y^{m}$, and for $d=2 m$ with $m$ odd, we still have extreme regular forms

$$
f_{0}=x^{(3 m+1) / 2} y^{(m-1) / 2}+x^{(m-1) / 2} y^{(3 m+1) / 2} .
$$

For $d \leq 10$ these are all regular extreme forms.

Summarizing the results for small values of $d$, there are only finitely many, up to a linear transformation, extreme exotic forms of degree $d<7$. For $d=6$ these are $x y^{5}, x^{2} y^{4}, x(x+y) y^{4}$. For $d=7, x(x+y)(x+a y) y^{4}$, $a \in K$, is an infinite family of exotic extreme linearly distinct forms.

TheOREM 3. For a binary exotic form $f$ of degree $d$ let

$$
\mathcal{D}_{f}=L_{1}^{e_{1}+1} \ldots L_{k}^{e_{k}+1} L_{k+1} \ldots L_{k+t},
$$

where $k \geq 1, e_{1}, \ldots, e_{k} \geq 1$, and $L_{i}$ are projectively distinct. Let $l_{i}$, for $i=k+1, \ldots, k+t$, be a non-zero linear form annihilated by $L_{i}$. Then there exist $a_{1}, \ldots, a_{t} \in K \backslash\{0\}$ such that

$$
f+a_{1} l_{k+1}^{d}+\cdots+a_{t} l_{k+t}^{d}=f_{0},
$$

where $f_{0}$ is an extreme form with

$$
\mathcal{D}_{f_{0}}=L_{1}^{e_{1}+1} \ldots L_{k}^{e_{k}+1} .
$$

Moreover, $f_{0}$ is the only extreme form such that $f \preceq f_{0}$ and the summands in the above decomposition of $f_{0}$ are uniquely determined by $f$.

It follows from the above theorem that if for a form $f$ we denote by $f^{-}$(by $f^{+}$) the partially ordered (by $\preceq$ ) set composed of all forms $g \preceq f$ ( $f \preceq g$, resp.), then $f^{+}$for all exotic forms $f$, and $f^{-}$for all plain forms, are isomorphic to a partially ordered (by inclusion) family of all subsets of a finite set.

TheOrem 4. Let $f$ be a regular form of degree $d=2 m$. Let

$$
\mathcal{D}_{1}=L_{1}^{e_{1}+1} \ldots L_{k}^{e_{k}+1} L_{k+1} \ldots L_{k+t}, \quad e_{1}+\cdots+e_{k}+k+t=m+1,
$$

where $k \geq 1, e_{1}, \ldots, e_{k} \geq 1$, and $L_{i}$ are projectively distinct. Let $l_{i}$, for $i=k+1, \ldots, k+t$, be a non-zero linear form annihilated by $L_{i}$.

If $\mathcal{D}_{1} f=0$, then there exist $a_{1}, \ldots, a_{t} \in K \backslash\{0\}$ such that

$$
f+a_{1} l_{k+1}^{d}+\cdots+a_{t} l_{k+t}^{d}=f_{0},
$$

where $f_{0}$ is an extreme form with

$$
\mathcal{D}_{f_{0}}=L_{1}^{e_{1}+1} \ldots L_{k}^{e_{k}+1} \text {. }
$$

The summands in the above decomposition of $f_{0}$ are uniquely determined by $f$. Moreover, there are only finitely many extreme forms $f_{0}$ such that $f \preceq f_{0}$, and every such form is defined in the way described above by some differential operator $\mathcal{D}_{1}$. 
Proposition 1. For a binary exotic form $f$ of degree $d$, a linear operator $L$ divides $\mathcal{D}_{f}$ if and only if $a l^{d}$, where $L l=0$ and $a \in K \backslash\{0\}$, does not appear as a summand in any presentation of $f$ of length $r(f)$.

The above proposition describes some obstacles for a sum $f=l_{1}^{d}+\cdots+l_{r}^{d}$ to appear in a minimal presentation of an extreme form $f_{0}$ given as

$$
f_{0}=g_{1} m_{1}^{d-e_{1}}+\cdots+g_{u} m_{u}^{d-e_{u}},
$$

where $m_{1}, \ldots, m_{u}$ are pairwise projectively distinct linear forms, $g_{1}, \ldots, g_{u}$ are forms of positive degree $e_{1}, \ldots, e_{u}$, respectively, and $m_{i} \nmid g_{i}$. The next proposition shows that if $r \leq r\left(f_{0}\right)-\lceil(d+2) / 2\rceil$, this is the only obstacle.

Proposition 2. Let $f$ be a plain binary form and let $f=l_{1}^{d}+\cdots+l_{r}^{d}$ be its presentation of minimal length. Let $f_{0}$ be an extreme form

$$
f_{0}=g_{1} m_{1}^{d-e_{1}}+\cdots+g_{u} m_{u}^{d-e_{u}}, \quad e_{1}+\cdots+e_{u}+u<(d+2) / 2,
$$

where $m_{1}, \ldots, m_{u}$ are pairwise projectively distinct linear forms, $g_{1}, \ldots, g_{u}$ are forms of positive degree $e_{1}, \ldots, e_{u}$, respectively, and $m_{i} \nmid g_{i}$. Assume that every linear form $l_{i}$ is projectively distinct from any form $m_{j}$, for $i=1, \ldots, r$, $j=1, \ldots, u$.

If $r \leq r\left(f_{0}\right)-\lceil(d+2) / 2\rceil$, then $f \prec f_{0}$. Moreover, for every degree $d>1$ the inequality is best possible.

COROLlary 4. For every form $f$ of degree $d$ with $r(f)<\lfloor d / 2\rfloor$ there exist infinitely many extreme forms $f_{0}$ such that $f \prec f_{0}$.

However, Corollary 4 is not best possible, as shown by

Proposition 3. For a given form $f$ of degree $d$, there exist infinitely many extreme forms $f_{0} \succ f$ if and only if $r(f) \leq(d+1) / 2$.

COROLlary 5. For every binary form $f$ exactly one of the inequalities $f \prec g, f \succ g$ admits infinitely many solutions $g$.

In fact, it follows from the above results that for all plain forms and regular forms of odd degree there exist only finitely many forms less than the given form, and infinitely many greater forms. For all exotic forms and for regular forms of even degree there exist infinitely many forms that are less than the given form, and only finitely many greater forms.

The next proposition describes a method for finding some extreme form greater than a given plain form.

Proposition 4. Let $f$ be a form with $r=r(f) \leq(d+1) / 2$. Let $G$ be the maximal squareful factor of $\mathcal{E}_{f} \in A_{d-r+2}(f)$. If $0<\operatorname{deg} G<(d+2) / 2$, then there exists an extreme form $f_{0}$ such that $\mathcal{D}_{f_{0}}=G$ and $f \prec f_{0}$. Conversely, if $f \prec f_{0}$, where $f_{0}$ is extreme, then $\mathcal{D}_{f_{0}}$ is a maximal squareful factor of some $\mathcal{E}_{f} \in A_{d-r+2}(f)$. 
Regular extreme forms $f$ are mysterious. We already know from Theorem 1 that they are of even degree $d$ and in case $d=2 m$, we have $r(f)=m+1$ and every element in $A_{m+1}(f)$ is either squarefree or squareful. The next theorem implies that up to a linear transformation, unexpectedly, there are only finitely many extreme regular forms of any even degree $d$, but their number, in general, is not explicitly given.

TheOREm 5. A regular form $f$ of degree $2 m$ is extreme if and only if either it is equivalent via a linear transformation to $x^{m} y^{m}$, or in $A_{m+1}(f)$ there are three coprime squareful forms that together have exactly $m+3$ projectively distinct linear factors.

Corollary 6. For $m \leq 5$ a regular form $f$ of degree $2 m$ is extreme if and only if either $f$ is equivalent via a linear transformation to $x^{m} y^{m}$, or $m=3$ or 5 and $f$ is equivalent via a linear transformation to $x^{(3 m+1) / 2} y^{(m-1) / 2}$ $-x^{(m-1) / 2} y^{(3 m+1) / 2}$.

COROllary 7. For every $m$ there exist up to a linear transformation only finitely many extreme regular forms of degree $2 \mathrm{~m}$.

Corollary 6 does not extend to $m>5$. Indeed, if $m=2 k l-1(l>1)$, there is an extreme regular form $f$ of degree $2 m$ with three squareful forms in $A_{m+1}(f)$ :

$$
\left(\left(X^{k}+Y^{k}\right)^{l}+Y^{k l}\right)^{2}, \quad\left(\left(X^{k}+Y^{k}\right)^{l}-Y^{k l}\right)^{2}, \quad Y^{k l}\left(X^{k}+Y^{k}\right)^{l} .
$$

For $k=1$ we obtain forms $f$ mentioned in Corollary 6, but for $k>1$ the explicit descriptions of the relevant forms $f$ are much more complicated, though, in any case, such a form is uniquely, up to projective equality, determined by two explicitly given differential equations. For $m=6$, J. Browkin has found an interesting explicit example given at the end of the paper.

In [1], the current authors considers presentations of a given form $f$ of degree $d$ in $n$ variables as a sum of powers $l_{1}^{d}+\cdots+l_{u}^{d}$, where $l_{1}, \ldots, l_{u}$ are linear forms and $l_{1}, \ldots, l_{u}$ do not belong to a given finite family of hyperplanes in the space of linear forms. This has led to the following definition:

Definition. One says that $f$ has a lot of presentations of length $r$ if for any finite set of points $\left\{p_{1}, \ldots, p_{m}\right\}$ in $K^{2} \backslash\{0\}$ there is a presentation $f=l_{1}^{d}+\cdots+l_{r}^{d}$ such that $l_{i}\left(p_{j}\right) \neq 0$ for all $i \leq r, j \leq m$.

For $f$ essentially depending on $n$ variables, let $\bar{r}(f)$ be the minimum $r$ such that $f$ has a lot of presentations of length $r$. In the case of binary forms $f$ essentially depending on two variables, it has been noticed in [1] that $\bar{r}(f) \leq d$ and $\bar{r}\left(x y^{d-1}\right)=d$. The next proposition determines $\bar{r}(f)$ for various forms $f$. 


\section{Proposition 5.}

1. For every exotic form $f, \bar{r}(f)=r(f)$.

2. For every plain form $f, \bar{r}(f)=d-r(f)+2$.

3. For every regular form $f$,

$$
\bar{r}(f)= \begin{cases}d / 2+1 & \text { for d even } \\ (d+3) / 2 & \text { for d odd }\end{cases}
$$

\section{Proofs of Theorems 1 and $1^{\prime}$}

LEMMA 3.1. For

$$
f=\sum_{i=0}^{d}\left(\begin{array}{l}
d \\
i
\end{array}\right) a_{i} x^{d-i} y^{i}, \quad d=2 n+1,
$$

let

$$
C(f)=\left|\begin{array}{cccc}
x^{n+1} & -x^{n} y & \ldots & \pm y^{n+1} \\
a_{n+1} & a_{n} & \ldots & a_{0} \\
a_{n+2} & a_{n+1} & \ldots & a_{1} \\
\ldots \ldots \ldots \ldots \ldots \ldots \ldots & \ldots \ldots \\
a_{2 n+1} & a_{2 n} & \ldots & a_{n}
\end{array}\right| .
$$

If $r(f) \leq n$, then $C(f)=0$. If $r(f)=n+1$ and

$$
f=\sum_{i=1}^{n+1} l_{i}^{d}
$$

where $l_{i}$ are projectively distinct non-zero linear forms, i.e. (1) is a presentation of $f$, then

$$
C(f)=k \prod_{i=1}^{n+1} l_{i},
$$

where $k \in K$ and the presentation is unique up to the order of summands.

Proof. See [5].

Lemma 3.2. If $r(f)>n$ and $\operatorname{discr} C(f)=0$, then $r(f)>n+1$.

Proof. If we had $r(f)=n+1$, then it would follow from (1) that

$$
\operatorname{discr} C(f) \neq 0 \text {. }
$$
then

LEMma 3.3. If $f=\sum_{i=1}^{n+1} l_{i}^{d}$ is a presentation of length $n+1=r(f)$,

$$
r\left(f+(a x+b y)^{d}\right) \leq n
$$

if and only if $(a x+b y)^{d}=-l_{j}^{d}$ for some $j \leq n+1$. 
Proof. Assume that (3) holds and

$$
f+(a x+b y)^{d}=\sum_{j=1}^{s} m_{j}^{d}
$$

is a presentation of length $s \leq n$. Then

$$
f=-(a x+b y)^{d}+\sum_{j=1}^{s} m_{j}^{d}
$$

and $s+1 \geq n+1$, hence $s=n, a x+b y$ is projectively distinct from $m_{j}$, and by uniqueness of presentation (Lemma 3.1)

$$
-(a x+b y)^{d}=l_{j}^{d}
$$

for some $j \leq n+1$. Conversely, if the above equality holds, then

$$
f+(a x+b y)^{d}=\sum_{i=1, i \neq j}^{n+1} l_{i}^{d}
$$

and (3) holds.

LEMma 3.4. If a form $f$ of degree $d>1$ is extreme, then $r(f)>(d+1) / 2$, i.e. $f$ is exotic or regular of even degree.

Proof. Assume that $f$ is extreme, $r=r(f)$ and let

$$
f=l_{1}^{d}+\cdots+l_{r}^{d}
$$

be an $r$-presentation. For every linear form $l \in K[x, y]$, we have

$$
f+l^{d}=m_{1}^{d}+\cdots+m_{s}^{d}
$$

for some linear forms $m_{1}, \ldots, m_{s}$ and $s \leq r$. Then

$$
l_{1}^{d}+\cdots+l_{r}^{d}+l^{d}=m_{1}^{d}+\cdots+m_{s}^{d}
$$

and this gives a presentation of zero of length at most $r+1+s$. Hence, by [1. Corollary 3],

$$
r+1+s \geq d+2 \text { and } 2 r+1 \geq d+2, \quad \text { i.e. } \quad r \geq(d+1) / 2 .
$$

It remains to exclude the case $d=2 n+1, r(f)=n+1$. Let $F=f+(a x+b y)^{d}$. For $d>1$, we have

$$
\operatorname{discr} C(F)=P \in K[a, b] .
$$

If $f=\sum_{i=1}^{n+1}\left(a_{i} x+b_{i} y\right)^{d}$ then by Lemmas 3.1 and 3.3 .

$$
P\left(-a_{i},-b_{i}\right)=0, \quad P(0,0) \neq 0,
$$

hence $P(a, b)=0$ defines a curve. This curve contains a point $\left(a_{0}, b_{0}\right)$ different from

$$
\left(-\zeta_{d}^{j} a_{i},-\zeta_{d}^{j} b_{i}\right) \quad(1 \leq i \leq n+1,0<j<d)
$$


and the form $F_{0}=f+\left(a_{0} x+b_{0} y\right)^{d}$ satisfies the assumptions of Lemma 3.2 hence $r\left(F_{0}\right)=n+1$ and $f$ is not extreme.

Lemma 3.5. Let $s(f)$ be defined as in Lemma 2.2. If $s(f) \leq(d+1) / 2$ and $\mathcal{D}_{f}$ is not squarefree, then $r(f)=d+2-s(f)$ and $\mathcal{E}_{f}$ can be chosen to be squarefree. If $s(f)=(d+2) / 2$, then $r(f)=s(f)$.

Proof. Let $r=r(f)$ and $s=s(f)$. By Lemma 2.1. $A_{r}(f)$ contains a squarefree form $G$. If $s \leq(d+1) / 2$, then by Lemma 2.2 (ii), $G=A \mathcal{D}_{f}+B \mathcal{E}_{f}$, where $A, B \in K[X, Y]$. Since $\mathcal{D}_{f}$ is not squarefree, we have $B \neq 0$, hence

$$
r=\operatorname{deg} G \geq \operatorname{deg} \mathcal{E}_{f}=d+2-s(f) .
$$

However, again by Lemma 2.2 we have $\left(\mathcal{D}_{f}, \mathcal{E}_{f}\right)=1$. Let $D_{3}$ be any form of degree $d+2-2 s(f)$ prime to $\mathcal{E}_{f}$. By Lemma 2.3 there are $a, b \in K$ such that $G_{a, b}=a \mathcal{D}_{f} D_{3}+b \mathcal{E}_{f}$ is squarefree. Hence $r=d+2-s(f)$ by Lemma 2.1 and since $b \neq 0$ we can replace $\mathcal{D}_{f}$ as one of the generators of $A(f)$ by $G_{a, b}$.

If $s=(d+2) / 2$, then by Lemma 2.2(ii), $A(f)$ is generated by two coprime forms $\mathcal{D}_{1}, \mathcal{D}_{2}$ both of degree $s$. By Lemma 2.3 there is a squarefree linear combination $a \mathcal{D}_{1}+b \mathcal{D}_{2}$ and this is a squarefree form of the least degree in $A(f)$. By Lemma 2.1, $r(f)=s$.

Lemma 3.6. If $f$ is exotic then $s(f) \leq(d+1) / 2, \mathcal{D}_{f}$ is not squarefree and

$$
A_{r-1}=\mathcal{D}_{f} K[X, Y]_{r-s-1} .
$$

Proof. If we had $s(f)=(d+2) / 2$, then by Lemma 2.2, $\mathcal{D}_{f}$ and $\mathcal{E}_{f}$ would be both of degree $s(f)$ and since $\left(\mathcal{D}_{f}, \mathcal{E}_{f}\right)=1, A(f)$ would contain, by Lemma 2.3, a squarefree operator of degree $(d+2) / 2$, hence by Lemma 2.1 we should have $r(f) \leq(d+2) / 2$, contrary to the assumption. If $\mathcal{D}_{f}$ were squarefree, we should have $r \leq s \leq(d+1) / 2$, a contradiction again. Finally, $A_{r-1}(f)$ does not contain any element $A \mathcal{D}_{f}+B \mathcal{E}_{f}$ with $B \neq 0$.

Proofs of Theorems 1 and $1^{\prime}$. We shall first prove that the condition is necessary. So assume that $f$ is extreme. Then by Lemma 3.4 we have

$$
r=r(f) \geq(d+2) / 2, \quad s=s(f)=d+2-r .
$$

Suppose that $A_{s}(f)$ contains an element $D$ which is neither squarefree nor squareful. Hence $D=D_{0} L$, where $L$ is a linear and $L$ does not divide $D_{0}$. Choose a linear form $l \neq 0$ such that $L l=0$.

Since $L D_{0} f=0$ we have

$$
D_{0} f=c_{1} l^{d-s+1} \quad \text { for some } c_{1} \in K .
$$

Since $L$ does not divide $D_{0}$,

$$
D_{0} l^{d}=c_{2} l^{d-s+1} \quad \text { for some } c_{2} \in K \backslash\{0\} .
$$

It follows that

$$
D_{0}\left(f-a l^{d}\right)=0 \quad \text { for } a=c_{1} / c_{2},
$$


and since $D_{0}$ of degree $s-1 \leq d / 2$ is not squarefree,

$$
\mathcal{D}_{f-a l^{d}}=D_{0}, \quad r\left(f-a l^{d}\right)=d+2-\operatorname{deg} D_{0}=d+3-s=r(f)+1 .
$$

The contradiction obtained shows that all elements of $A_{s}(f)$ are either squarefree or squareful. If $f$ is exotic, then, by Lemma 2.1. $A_{s}(f)$ contains no squarefree elements, hence all elements there are squareful.

In order to prove that the condition is sufficient, assume that

$$
r=r(f) \geq(d+2) / 2, \quad s=s(f)=d+2-r
$$

and that all elements of $A_{s}(f)$ are either squarefree or squareful. Let $l \in$ $K[x, y]$ be a linear form and choose a linear operator $L \neq 0$ such that $L l=0$. Suppose that

$$
r\left(f+l^{d}\right)=r+1 .
$$

Then by Lemma 3.5 ,

$$
s\left(f+l^{d}\right)=d+2-r-1=d+1-r \leq d / 2
$$

and for $D_{1}=\mathcal{D}_{f+l^{d}}$ we have

$$
D_{1} f+D_{1} l^{d}=0 .
$$

It follows that

$$
L D_{1} f=-L D_{1} l^{d}=-D_{1} L l^{d}=0, \quad L D_{1} \in A_{s}(f) .
$$

By the assumption, $L D_{1}$ is either squarefree or squareful. If $L D_{1}$ is squarefree then $D_{1}$ is squarefree, contrary to Lemma 3.6. Therefore $L D_{1}$ is squareful, $L$ divides $D_{1}$,

$$
D_{1} f=-D_{1} l^{d}=0
$$

and by Lemma 3.5 .

$$
r(f)=d+2-\operatorname{deg} D_{1}=r+1,
$$

a contradiction. This completes the proof of Theorem 1 . Theorem $1^{\prime}$ follows now from the last statement of Lemma 3.6.

Proof of Corollary 1. We can assume without loss of generality that the linear factor in question is $y$. Then $\mathcal{D}_{f} \mid X^{d-m+1}$ and, since $\mathcal{D}_{f}$ does not divide $X$ for $m<d, \mathcal{D}_{f}$ is squareful.

Proof of Corollary 2. If $d \geq 5, r(f) \geq d-1$ and $f$ is extreme, then by Lemma 3.5, $s=s(f) \leq 3$, and by Theorem 1, $\mathcal{D}_{f}=L^{s}$, where $L$ is a linear operator. Assuming without loss of generality that $L=Y$ we obtain

$$
f=\sum_{i=0}^{s-1} a_{i} x^{d-i} y^{i}, \quad a_{s-1} \neq 0,
$$

and $f$ has a factor $x$ with multiplicity $d-s+1=r+1$. 
To prove the second assertion of the corollary put $f=x^{d-1} y+x y^{d-1}$. If $d \geq 7$, we have $\mathcal{D}_{f}=X^{2} Y^{2}$ and Theorem 1 applies. If $d=6$ every element of $A_{4}(f)$ is of the form $a\left(X^{4}-Y^{4}\right)+b X^{2} Y^{2}$ and is either squarefree, if $a\left(b^{2}+4 a^{2}\right) \neq 0$, or squareful otherwise, and Theorem 1 applies.

4. Proof of Theorem 2, Assume that $f$ is extreme and exotic. Then it follows from Theorem $1^{\prime}$ that $\mathcal{D}_{f}$ is squareful and

$$
\mathcal{D}_{f}=M_{1}^{e_{1}+1} \ldots M_{u}^{e_{u}+1},
$$

where $e_{i} \geq 1$ for $i=1, \ldots, u$ and $e_{1}+\cdots+e_{u}+u<(d+2) / 2$. For $i=1, \ldots, u$, let $m_{i}$ be a non-zero linear form such that $M_{i} m_{i}=0$. Then it follows from Lemma 2.1 that

$$
f=g_{1} m_{1}^{d-e_{1}}+\cdots+g_{u} m_{u}^{d-e_{u}},
$$

where $g_{i}, e_{i}$ satisfy the above conditions. Thus the conditions given in the propositions for a form to be extreme and exotic are necessary.

In order to prove that they are also sufficient, assume that $f$ can be decomposed as

$$
f=g_{1} m_{1}^{d-e_{1}}+\cdots+g_{u} m_{u}^{d-e_{u}}
$$

with $g_{i}, e_{i}$ and $m_{i}$ as above. Let $M_{i} m_{i}=0$ for some non-zero linear operators $M_{i}$, where $i=1, \ldots, u$. Then

$$
M_{i}^{e_{i}+1} g_{i} m_{i}^{d-e_{i}}=m_{i}^{d-e_{i}}\left(M_{i}^{e_{i}+1} g_{i}\right)=0,
$$

since

$$
\operatorname{deg}\left(M_{i}^{e_{i}+1} g_{i}\right)=e_{i}+1>e_{i}=\operatorname{deg} g_{i} .
$$

Hence $f$ is annihilated by $M_{1}^{e_{1}+1} \ldots M_{u}^{e_{u}+1}$. On the other hand,

$$
M_{i}^{e_{i}} g_{i} m_{i}^{d-e_{i}}=m_{i}^{d-e_{i}}\left(M_{i}^{e_{i}} g_{i}\right)=a_{i} m_{i}^{d-e_{i}} \neq 0,
$$

where $a_{i} \in K \backslash\{0\}\left(g_{i}\right.$ is not divisible by $m_{i}$, hence $\left.M_{i}^{e_{i}} g_{i} \neq 0\right)$ and

$$
\begin{aligned}
M_{i}^{e_{i}}\left(\prod_{j=1, j \neq i}^{u} M_{j}^{e_{j}+1}\right) f & =\left(\prod_{j=1, j \neq i}^{u} M_{j}^{e_{j}+1} M_{i}^{e_{i}}\right) g_{i} m_{i}^{d-e_{i}} \\
& =\prod_{j=1, j \neq i}^{u} M_{j}^{e_{j}+1} a_{i} m_{i}^{d-e_{i}}=b_{i} m_{i}^{d-\left(e_{1}+\cdots+e_{u}+u-1\right)} \neq 0 .
\end{aligned}
$$

The last inequality follows from $d-\left(e_{1}+\cdots+e_{u}+u-1\right)>0, M_{j} m_{i} \neq 0$ for $j=1, \ldots, u, j \neq i$. Thus $f$ is annihilated by

$$
D=\prod_{i=1}^{u} M_{i}^{e_{i}+1}
$$

but, for $i=1, \ldots, u, f$ is not annihilated by $D / M_{i}$. Thus $D=\mathcal{D}_{f}$. Now, 
it follows from Theorem $1^{\prime}$ that $f$ is extreme and exotic with $r(f)=d-$ $\operatorname{deg} \mathcal{D}_{f}+2=d-\left(e_{1}+\cdots+e_{u}+u\right)+2>(d+2) / 2$.

\section{Proofs of Theorems 3 and 4}

Lemma 5.1. Let $r=r(f) \geq(d+2) / 2$, and let $L_{i}$ for $1 \leq i \leq k+t$ denote projectively distinct linear operators. If $t \geq 1$ and

$$
\mathcal{D}_{f}=L_{1}^{e_{1}+1} \ldots L_{k}^{e_{k}+1} L_{k+1} \ldots L_{k+t},
$$

then for some $a_{t} \neq 0, f_{1}=f+a_{t} l_{k+t}^{d}$ has the minimal length of presentation equal to $r+1$ and

$$
\mathcal{D}_{f_{1}}=L_{1}^{e_{1}+1} \ldots L_{k}^{e_{k}+1} L_{k+1} \ldots L_{k+t-1} .
$$

Proof. Let $L_{i} l_{i}=0$ for $1 \leq i \leq k+t$ and

$$
H=L_{1}^{e_{1}+1} \ldots L_{k}^{e_{k}+1} L_{k+1} \ldots L_{k+t-1} .
$$

Then $H f \neq 0$, but $L_{k+t} H f=0$. Hence $H f=a l_{k+t}^{d-s+1}$ for some $a \in K \backslash\{0\}$ and $s=d+2-r$. Thus

$$
H\left(f+a_{t} l_{k+t}^{d}\right)=0,
$$

where $a_{t}$ is chosen in such a way that $H\left(a_{t} l_{k+t}^{d}\right)=-a l_{k+t}^{d-s+1}$. Since deg $H=$ $s-1$ it follows from Lemma 3.5 that

$$
r\left(f+a_{t} l_{t}^{d}\right) \geq d-(s-1)+2=r+1 .
$$

Since obviously $r\left(f+a_{t} l_{t}^{d}\right) \leq r+1$ we have $r\left(f+a_{t} l_{t}^{d}\right)=r+1$ and $s\left(f+a_{t} l_{t}^{d}\right)=$ $s-1=\operatorname{deg} H$. In view of (4) we have

$$
H=\mathcal{D}_{f+a_{t} l_{k+t}^{d}}=\mathcal{D}_{f_{1}} .
$$

Proof of Theorem 3. It follows from Lemma 5.1, by induction on $t$, that there exist $a_{1}, \ldots, a_{t}$ in $K \backslash\{0\}$ such that

$$
f+a_{1} l_{k+1}^{d}+\cdots+a_{t} l_{k+t}^{d}=f_{0},
$$

where $f_{0}$ is an extreme form with $\mathcal{D}_{f_{0}}=L_{1}^{e_{1}} \ldots L_{k}^{e_{k}}$. The minimal length of presentation of $f_{0}$ is equal to $d-(s-t)+2=r+t$. Thus

$$
f_{0}-f=a_{1} l_{k+1}^{d}+\cdots+a_{t} l_{k+t}^{d}
$$

is a presentation of minimal length and hence it is unique for fixed $f_{0}$, since its length is $t<s-1<d / 2$.

It suffices to show that $f_{0}$ is unique. Notice that anyway

$$
L_{1}^{e_{1}+1} \ldots L_{k}^{e_{k}+1}\left(f_{0}\right)=0
$$

and

$$
\begin{aligned}
L_{k+1} \ldots L_{k+t}\left(f_{0}\right) & =L_{k+1} \ldots L_{k+t}\left(f+\left(a_{1} l_{k+1}^{d}+\cdots+a_{t} l_{k+t}^{d}\right)\right) \\
& =L_{k+1} \ldots L_{k+t} f \neq 0 .
\end{aligned}
$$


If $f_{0}, f_{0}^{\prime}$ both satisfy these conditions, then

$$
L_{k+1} \ldots L_{k+t}\left(f_{0}-f_{0}^{\prime}\right)=0,
$$

hence $f_{0}-f_{0}^{\prime}$, annihilated by $\mathcal{D}_{f_{0}}=\mathcal{D}_{f_{0}^{\prime}}$, can be represented, by Lemma 2.1 . as a sum of powers of linear forms projectively equal to $l_{k+1}, \ldots, l_{k+t}$. This is not possible unless $f_{0}-f_{0}^{\prime}=0$, because both operators annihilating $f_{0}-f_{0}^{\prime}$, namely $\mathcal{D}_{f_{0}}$ and $L_{k+1} \ldots L_{k+t}$, have degrees smaller than $(d+2) / 2$, but are relatively prime.

Proof of Theorem 4. For the proof of the first part of the theorem it suffices to use the same argument as in the proof of Theorem 3. Finiteness of the set of extreme forms $f_{0}$ such that $f \preceq f_{0}$ follows from Lemma 2.3 .

6. Proof of Proposition 1 1 . In order to prove that the condition for $L \mid \mathcal{D}_{f}$ is necessary, let $l$ be a non-zero linear form such that $L l=0$. If $a l^{d}$ appears in a presentation of $f$ of length $r=r(f)$, then $A_{r}(f)$ contains a squarefree operator $D$ of degree $r$ divisible by $L$. By Lemma 2.2 we have $D=\mathcal{D}_{f} A+\mathcal{E}_{f} B$, where $A, B$ are forms from $K[X, Y]$. Since $\operatorname{deg} D=\operatorname{deg} \mathcal{E}_{f}$, we have $B \in K$, and $\left(\mathcal{D}_{f}, \mathcal{E}_{f}\right)=1$ gives $B=0$. Thus $D$ divisible by $\mathcal{D}_{f}$ is not squarefree, a contradiction.

In the opposite direction assume that $L$ does not divide $\mathcal{D}_{f}$ and that $L l=0$. Now, if $\operatorname{deg} H<r$ and

$$
H L f=H(L f)=0,
$$

then $\mathcal{D}_{f} \mid H L$, but since $L$ does not divide $\mathcal{D}_{f}$, we have $\mathcal{D}_{f} \mid H$. On the other hand, $\mathcal{D}_{f} L f=0$. Thus if $s(L f) \leq d / 2$, then $\mathcal{D}_{L f}=\mathcal{D}_{f}$ and by Lemma 3.5 .

$$
r(L f)=\operatorname{deg} L f+2-\operatorname{deg} \mathcal{D}_{L f}=d+1-(d+2-r(f))=r-1 .
$$

If $s(L f)=(d+1) / 2$, then again

$$
r(L f)=d+1-s(L f)=(d+2-r(f))=(d+1) / 2=r-1 .
$$

Let

$$
L f=l_{1}^{d-1}+\cdots+l_{r-1}^{d-1} .
$$

If $l$ is not projectively equal to any of $l_{i}$, then integrating this presentation with respect to $L$ we obtain a form

$$
f_{0}=a_{1} l_{1}^{d}+\cdots+a_{r-1} l_{r-1}^{d}, \quad a_{1}, \ldots, a_{r-1} \in K,
$$

of degree $d$ such that $f-f_{0} \neq 0$ is annihilated by $L$, hence equal to $a l^{d}$, $a \neq 0$. Thus we have a presentation of length $r$

containing $a l^{d}$.

$$
f=a_{1} l_{1}^{d}+\cdots+a_{r-1} l_{r-1}^{d}+a l^{d}
$$

If $l$ is projectively equal to $l_{i}$, then $A_{r-1}(f)$ has a squarefree element $G_{0}$ divisible by $L$. Let $A_{0}$ be an element of $K[X, Y]_{2 r-d-3}$ not divisible by $L$. 
Since $G_{0}$ is squarefree, the discriminant of $a A_{0} \mathcal{D}_{f}+b G_{0}$ is not identically 0 in $a, b$. Hence there exist $a_{0} \in K \backslash\{0\}$ and $b_{0} \in K$ such that

$$
G_{1}=a_{0} \mathcal{D}_{f}+b_{0} G_{0}
$$

is squarefree. We have $G_{1} \in A_{r-1}(L f)$ and $L$ does not divide $G_{1}$, hence by Lemma 2.1. $L f$ has a presentation (5) such that no $l_{i}$ is projectively equal to $l$ and this case has been considered earlier.

7. Proofs of Proposition 2 and of Corollary 4. First we prove the following

Lemma 7.1. The formula

$$
\sum_{i=1}^{d} \frac{\left(x+z_{i} y\right)^{d}}{\prod_{j=1, j \neq i}^{d}\left(z_{i}-z_{j}\right)}=d x y^{d-1}
$$

for distinct $z_{i}$ satisfying

$$
z_{1}+\cdots+z_{d}=0
$$

gives all presentations of $d x y^{d-1}$ of the minimal length $d$.

Proof. Since $X^{2}$ annihilates $d x y^{d-1}$, by Lemma 3.5 the minimal length of a presentation of $d x y^{d-1}$ is $d$. By Proposition 1 every form in such a presentation depends essentially on $x$. An identity

$$
\sum_{j=1}^{d} a_{j}\left(x+z_{j} y\right)^{d}=d x y^{d-1}
$$

implies that

$$
\sum_{j=1}^{d} a_{j} z_{j}^{i}=0 \quad(i=0, \ldots, d-2, d), \quad \sum_{j=1}^{d} a_{j} z_{j}^{d-1}=1,
$$

hence

$$
\left|\begin{array}{cccc}
1 & \ldots & 1 & 0 \\
z_{1} & \ldots & z_{d} & 0 \\
\ldots \ldots \ldots \ldots & \ldots \ldots \\
z_{1}^{d-2} & \ldots & z_{d}^{d-2} & 0 \\
z_{1}^{d-1} & \ldots & z_{d}^{d-1} & 1 \\
z_{1}^{d} & \ldots & z_{d}^{d} & 0
\end{array}\right|=-\left|\begin{array}{ccc}
1 & \ldots & 1 \\
z_{1} & \ldots & z_{d} \\
\ldots \ldots \ldots \ldots \ldots . \\
z_{1}^{d-2} & \ldots & z_{d}^{d-2} \\
z_{1}^{d} & \ldots & z_{d}^{d}
\end{array}\right|=0
$$

and since $z_{j}$ are distinct, we have (7) by a well known formula (see [4, p. 333]) for the last determinant. The coefficients $a_{j}$ are determined by a system of equations obtained from (9) for $i=0, \ldots, d-1$. Using Cramer's formulae 
and the formula for the Vandermonde determinant we obtain

$$
a_{j}=\prod_{\substack{j=1 \\ j \neq i}}^{d}\left(z_{i}-z_{j}\right)^{-1},
$$

which gives (6). Conversely, if (7) and (11) are satisfied for distinct $z_{i}$, we obtain (10) and (9), hence (8).

Proof of Proposition 2. Let $L_{i}$ be non-zero linear operators such that

$$
L_{i} l_{i}=0 \quad \text { for } i=1, \ldots, r+1, \ldots, r+u .
$$

We shall prove below that

(*) $\quad f_{1}=-f+\left(g_{1} l_{r+1}^{d-e_{1}}+\cdots+g_{u} l_{r+u}^{d-e_{u}}\right)$ is either exotic and

$$
\mathcal{D}_{f_{1}}=L_{1} \ldots L_{r} L_{r+1}^{e_{1}+1} \ldots L_{r+u}^{e_{u}+1},
$$

or regular of even degree and

$$
L_{1} \ldots L_{r} L_{r+1}^{e_{r}+1} \ldots L_{r+u}^{e_{u}+1} \in A_{(d+2) / 2}(f) .
$$

Denote $e_{1}+\cdots+e_{u}$ by $e$. Notice that

$$
D=L_{1} \ldots L_{r} L_{r+1}^{e_{1}+1} \ldots L_{r+u}^{e_{u}+1}
$$

annihilates $f_{1}$ and has

$$
\begin{aligned}
\operatorname{deg} D & =r+e+u=r+d-r\left(f_{0}\right)+2 \\
& \leq r\left(f_{0}\right)-\lfloor(d+2) / 2\rfloor+d-r\left(f_{0}\right)+2 \\
& =d+2-\lfloor(d+2) / 2\rfloor=\lceil(d+2) / 2\rceil .
\end{aligned}
$$

By Lemma 3.5 and Theorem 2 , in order to prove $(*)$, it remains to show that no proper factor of $D$ annihilates $f_{1}$. Hence it remains to show that $L_{i}^{-1} D f_{1} \neq 0$ for $i=1, \ldots, r+u$. For $i=1, \ldots, r$, this is clear:

$$
L_{i}^{-1} D f_{1}=c_{i} l_{i}^{d-e-u-r+1}, \quad c_{i} \neq 0,
$$

since $L_{j} l_{i} \neq 0$ whenever $j \neq i$. For $i=r+1, \ldots, r+u$, we have

$$
\begin{aligned}
L_{i}^{-1} D f_{1} & =L_{i}^{-1} D g_{i-r} l_{i}^{d-e_{i-r}} \\
& =\left(L_{i}^{-e_{i-r}-1} D\right) L_{i}^{e_{i-r}} g_{i-r} l_{i}^{d-e_{i-r}}=a_{i}\left(L_{i}^{-e_{i-r}-1} D\right) l_{i}^{d-e_{i-r}},
\end{aligned}
$$

where $a_{i} \in K \backslash\{0\}$, since $g_{i-r}$ is of degree $e_{i-r}$ and is not divisible by $l_{i}$. Then

$$
\left(L_{i}^{-e_{i-r}-1} D\right) l_{i}^{d-e_{i-r}}=b_{i} l_{i}^{d-e-u} \neq 0, \quad b_{i} \in K \backslash\{0\},
$$

since no $L_{j}$ with $j \neq i$ annihilates $l_{i}$.

Then we consider the decomposition of $f_{1}$ given by

$$
f_{1}=-l_{1}^{d}-\cdots-l_{r}^{d}+\left(g_{1} l_{r+1}^{d-e_{1}}+\cdots+g_{u} l_{r+u}^{d-e_{u}}\right) .
$$

We have $r\left(f_{1}\right)=d-(r+(e+u))+2$. Denote $r\left(f_{1}\right)$ by $r_{1}$. Let

$$
f_{1}=m_{1}^{d}+\cdots+m_{r_{1}}^{d}
$$


be a presentation of minimal length. Then

$$
g_{1} l_{r+1}^{d-e_{1}}+\cdots+g_{u} l_{r+u}^{d-e_{u}}=l_{1}^{d}+\cdots+l_{r}^{d}+m_{1}^{d}+\cdots+m_{r_{1}}^{d}
$$

is also of minimal length equal to $d-(e+u)+2$. Hence

$$
f=l_{1}^{d}+\cdots+l_{r}^{d} \preceq g_{1} l_{r+1}^{d-e_{1}}+\cdots+g_{u} l_{r+u}^{d-e_{u}},
$$

where $g_{1} l_{r+1}^{d-e_{1}}+\cdots+g_{u} l_{r+u}^{d-e_{u}}$ is extreme.

This completes the proof of the first part of Proposition 2. In order to prove the second part we proceed in the following way. Let $\zeta_{n}$ be a primitive root of unity of order $n$ and consider first $d=2 r$. If $r=1$ we have $x^{2} \nprec x y$, hence the inequality $r(f) \leq r\left(f_{0}\right)-2$ cannot be weakened. If $r>1$ we shall show that $f \nprec f_{0}$, where

$$
f=x^{2 r}+\sum_{i=2}^{r}\left(x+\zeta_{r-1}^{i} y\right)^{2 r}, \quad f_{0}=2(1-2\lfloor 2 / r\rfloor) r x y^{2 r-1} .
$$

Assuming the contrary we infer from Lemma 7.1 the existence of $z_{1}, \ldots, z_{2 r}$ in $K$ such that

$$
\begin{gathered}
z_{1}+\cdots+z_{2 r}=0, \\
z_{1}=0, \quad z_{i}=\zeta_{r-1}^{i} \quad(2 \leq i \leq r), \\
\prod_{\substack{j=1 \\
j \neq i}}^{2 r}\left(z_{i}-z_{j}\right)=(1-2\lfloor 2 / r\rfloor)^{-1}=1-2\lfloor 2 / r\rfloor .
\end{gathered}
$$

Now, (12) and (13) give

$$
z_{r+1}+\cdots+z_{2 r}=-\lfloor 2 / r\rfloor,
$$

while (13) and (14) give

$$
\begin{aligned}
\prod_{j=r+1}^{2 r}\left(-z_{j}\right) & =(1-2\lfloor 2 / r\rfloor) \prod_{j=2}^{r}\left(-\zeta_{r-1}^{j}\right)^{-1}=2\lfloor 2 / r\rfloor-1, \\
\prod_{j=r+1}^{2 r}\left(\zeta_{r-1}^{i}-z_{j}\right) & =(1-2\lfloor 2 / r\rfloor) \prod_{\substack{j=1 \\
j \neq i}}^{r}\left(\zeta_{r-1}^{i}-\zeta_{r-1}^{j}\right)^{-1} \\
& =(1-2\lfloor 2 / r\rfloor) \prod_{k=1}^{r-2}\left(1-\zeta_{r-1}^{k}\right)^{-1} \\
& =(1-2\lfloor 2 / r\rfloor)(r-1)^{-1} \quad(2 \leq i \leq r) .
\end{aligned}
$$

Denoting by $y_{k}$ the $(r-k)$ th elementary symmetric polynomial of $-z_{r+1}, \ldots$, $-z_{2 r}$ and using (11) we can write the above system of equations in the 
form

$$
\begin{aligned}
y_{0} & =2\lfloor 2 / r\rfloor-1, \\
\sum_{k=0}^{r-2} \zeta_{r-1}^{i k} y_{k} & =(1-2\lfloor 2 / r\rfloor)(r-1)^{-1}-\zeta_{r-1}^{i r}-\zeta_{r-1}^{i(r-1)}\lfloor 2 / r\rfloor .
\end{aligned}
$$

For $r=2$ we obtain an insoluble system of equations $y_{0}=1, y_{0}=-3$. For $r>2$ solvability of the system implies

$$
\mathcal{F}=\left|\begin{array}{ccccc}
1 & 0 & \ldots & 0 & -1 \\
1 & 1 & \ldots & 1 & (r-1)^{-1}-1 \\
\ldots \ldots \ldots & \ldots & \ldots \ldots \ldots \ldots \ldots \ldots \ldots \ldots \ldots \\
1 & \zeta_{r-1}^{r-2} & \ldots & \left(\zeta_{r-1}^{r-2}\right)^{r-2} & (r-1)^{-1}-\zeta_{r-1}^{r-2}
\end{array}\right|=0 .
$$

Adding the second column to the last one and then developing the determinant according to the first row we obtain

$$
\begin{aligned}
\mathcal{F} & =(r-1)^{-1} \prod_{i=0}^{r-2} \zeta_{r-1}^{i} \prod_{j>i}\left(\zeta_{r-1}^{j}-\zeta_{r-1}^{i}\right)+(-1)^{r} \prod_{j>i}\left(\zeta_{r-1}^{j}-\zeta_{r-1}^{i}\right) \\
& =(-1)^{r} \frac{r-2}{r} \prod_{j>i}\left(\zeta_{r-1}^{j}-\zeta_{r-1}^{i}\right) \neq 0,
\end{aligned}
$$

contrary to (16). This contradiction shows that $f \nprec f_{0}$.

Consider now $d=2 r+1$. If $r=1$, we have $(x+2 y)^{3} \nprec 27 x y^{2}$, since $(x+2 y)^{3}-27 x y^{2}$ has the factor $x-y$ with multiplicity 2 . Thus the inequality $r(f) \leq r\left(f_{0}\right)-3$ cannot be weakened.

If $r>1$, we shall show that $f \nprec f_{0}$, where

$$
\begin{aligned}
f & =\sum_{i=1}^{r}\left(x+\zeta_{r}^{i} r^{-1 / 2 r} y\right)^{2 r+1}=r x^{2 r+1}+r^{1 / 2}\left(\begin{array}{c}
2 r+1 \\
r
\end{array}\right) x^{r+1} y^{r}+\left(\begin{array}{c}
2 r+1 \\
2 r
\end{array}\right) x y^{2 r}, \\
f_{0} & =(2 r+1) x y^{2 r} .
\end{aligned}
$$

Assuming the contrary we infer from Lemma 7.1 the existence of distinct $z_{1}, \ldots, z_{2 r+1} \in K$ such that

$$
\begin{gathered}
z_{1}+\cdots+z_{2 r+1}=0, \\
z_{i}=\zeta_{r}^{i} r^{-1 / 2 r}, \quad \prod_{\substack{j=1 \\
j \neq i}}^{2 r+1}\left(z_{i}-z_{j}\right)=1 \quad(1 \leq i \leq r) .
\end{gathered}
$$

Now, (17) and (18) give 


$$
z_{r+1}+\cdots+z_{2 r+1}=0
$$

$$
\begin{aligned}
\prod_{j=r+1}^{2 r+1}\left(\zeta_{r}^{i} r^{-1 / 2 r}-z_{j}\right) & =\prod_{\substack{j=1 \\
j \neq i}}^{r}\left(\zeta_{r}^{i} r^{-1 / 2 r}-\zeta_{r}^{j} r^{-1 / 2 r}\right)^{-1} \\
& =r^{(r-1) / 2 r}\left(\zeta_{r}^{i}\right)^{1-r} \prod_{k=r-1}^{r-1}\left(1-\zeta_{r}^{k}\right)^{-1}=r^{-(r+1) / 2 r} \zeta_{r}^{i(r+1)},
\end{aligned}
$$

hence denoting by $y_{k}$ the $(r+1-k)$ th elementary symmetric polynomial of $-z_{r+1}, \ldots,-z_{2 r+1}$ and using (19) we obtain

$$
\zeta_{r}^{i(r+1)} r^{-(r+1) / 2 r}+\sum_{k=0}^{r-1}\left(\zeta_{r}^{r i} r^{-1 / 2 r}\right)^{k} y_{k}=\zeta_{r}^{i(r+1)} r^{-(r+1) / 2 r} .
$$

It follows from (19) and (20) that $y_{k}=0(0 \leq k \leq r)$, hence $z_{r+1}=$ $\cdots=z_{2 r+1}=0$, contrary to the condition that the $z_{i}$ are distinct. This contradiction shows that $f \nprec f_{0}$ and completes the proof.

Proof of Corollary 4. For $r \leq d-\lceil(d+2) / 2\rceil=\lfloor d / 2\rfloor-1$ we infer from Proposition 2 that the form $f=l_{1}^{d}+\cdots+l_{r}^{d}$, where $l_{i}$ are projectively distinct and not divisible by $y$, satisfies $f \prec x y^{d-1}$. Thus for any two linearly independent linear forms $l$ and $m$ such that $m \nmid l_{i}(1 \leq i \leq r)$ we have $f \prec l m^{d-1}$.

\section{Proofs of Propositions 3 and 4}

LEMMA 8.1. Let $d$ be odd, $u=(d+1) / 2$, and $f=\sum_{i=1}^{u} l_{i}^{d}$. Let $l^{d}$ be projectively different from all $l_{i}^{d}, i=1, \ldots, u$. For some $a \in K^{*}, f+a l^{d}$ admits a minimal presentation of length $u+1$ if and only if $A_{u+1}(f)$ contains an operator $D=D_{1} L D_{2}$, where $L l=0, D_{1}, D_{2} \in K[X, Y], D_{1}$ is squareful, $\operatorname{deg} D_{1}>0$ and $D_{1} D_{2}$ is not divisible by $L$.

Proof. Assume that $f+a l^{d}$ has a minimal presentation of length $u+1$. It is sufficient to consider the case $a=1$. Then $\mathcal{D}_{f+l^{d}}$ is of degree $u$ and $\mathcal{D}_{f+l^{d}}=D_{1} D_{2}$, where $D_{1}$ is squareful, $\operatorname{deg} D_{1}>0$ and

$$
\mathcal{D}_{f+l^{d}} f=\mathcal{D}_{f+l^{d}}\left(f+l^{d}\right)-\mathcal{D}_{f+l^{d}} l^{d}=0-c l^{d-u}=-c l^{d-u} .
$$

Thus

$$
D_{1} L D_{2} f=0
$$

and $D_{1} L D_{2} \in A_{u+1}(f)$. Moreover, since, by Proposition 1, no linear factor of $\mathcal{D}_{f+l^{d}}$ can annihilate a summand in the minimal presentation of $f+l^{d}$, it follows that $D_{1} D_{2}$ is not divisible by $L$.

Now, assume that $D_{1} L D_{2} \in A_{u+1}(f)$, where $D_{1}, D_{2}, L$ satisfy the assumptions of the lemma. Then $D_{1} D_{2}(f) \neq 0$ is annihilated by $L$ and hence $D_{1} D_{2} f=b l^{d-u}$ for some $b \in K^{*}$. Since $D_{1} D_{2}$ is not divisible by $L$, we have 
$D_{1} D_{2}\left(l^{d}\right) \neq 0$. Thus, for some $a \in K^{*}$, we have $D_{1} D_{2}\left(f+a l^{d}\right)=0$. Notice now that $f+a l^{d}$ admits a presentation of length $u+1$,

$$
f+a l^{d}=l_{1}^{d}+\cdots+l_{u}^{d}+a l^{d}
$$

and this presentation is of minimal length. In fact, $f+a l^{d}$ cannot have presentations of length less than $u$, since otherwise $f$ would admit two different presentations of length $u$ (only one of them containing $a l^{d}$ ). Hence the minimal length of presentation of $f+a l^{d}$ is at least $u$. If it were $u$, then $A\left(f+a l^{d}\right)_{u}$ would contain both $D_{1} D_{2}$ and a squarefree operator $\mathcal{D}_{f+a l}$. This is not possible, since $u=(d+1) / 2$.

Thus the minimal length of presentation of $f+a l^{d}$ is $u+1$. This completes the proof of the lemma.

Proof of Proposition 3. Necessity of the condition follows from Theorems 3 and 4 . In order to prove sufficiency, consider first the case where $f$ is plain. Let $f=l_{1}^{d}+\cdots+l_{u}^{d}$ be its presentation of minimal length. Since $f$ is plain, $2 u<d+1$. Let $l$ be a non-zero linear form projectively different from all $l_{1}, \ldots, l_{u}$ and let $g=f+l^{d}$. Then $g=l_{1}^{d}+\cdots+l_{u}^{d}+l^{d}$ is a presentation of minimal length. In fact, otherwise $g=l_{1}^{d}+\cdots+l_{u}^{d}+l^{d}=r_{1}^{d}+\cdots+r_{u}^{d}$ for some linear forms $r_{1}, \ldots, r_{u}$ and we would have

$$
l_{1}^{d}+\cdots+l_{u}^{d}+l^{d}-r_{1}^{d}-\cdots-r_{u}^{d}=0 .
$$

Since $l_{1}, \ldots, l_{u}, l$ are projectively different and $2 u+1 \leq d+1$, this is not possible by Lemma 5.3 of [1]. It follows that for any form $l$ as above, there exists an extreme form $h \succ f$ such that $l^{d}$ appears in a presentation of $h-f$ of minimal length. In fact, every extreme form $h \succ g \succ f$ has this property.

Now, if there exists a plain form which is dominated by only finitely many extreme forms, then there also exists such a plain form with minimal length of presentation equal to $u=\lfloor d / 2\rfloor$. Let us consider this case. If an extreme form $h$ dominates $f$, then $h-f$ is plain, since $h-f$ admits a presentation of length at most $r(h)-r(f) \leq d-u \leq d / 2<(d+1) / 2$. Thus the presentation of $h-f$ is unique and if the number of extreme forms dominating $f$ is finite, only a finite number of powers of linear forms can appear in all presentations of differences $h-f$, where $h \succ f$ and $h$ is extreme. This contradicts the result proved above saying that every form $l$ projectively different from $l_{1}, \ldots, l_{u}$ appears in a minimal presentation of some difference $h-f$, where $h$ is a properly chosen extreme form dominating $f$.

Consider the case where $f$ is regular of odd degree $d$. Then $f$ can be presented as $l_{1}^{d}+\cdots+l_{u}^{d}$, where $u=(d+1) / 2$, and the presentation is unique. Consider the space $A_{u+1}(f)$, of differential operators of degree $u+1$ annihilating $f$. Then

$$
A_{u+1}(f)=\left\{a \mathcal{D}_{f} X+b \mathcal{D}_{f} Y+c \mathcal{E}_{f}: a, b, c \in K\right\},
$$


hence is of dimension 3. Moreover $\mathcal{D}_{f}=L_{1} \ldots L_{u}$, where $L_{1}, \ldots, L_{u}$ are nonzero differential operators such that $L_{i} l_{i}=0$ for $i=1, \ldots, u$. Let $W$ be the subset of $A_{u+1}(f)$ corresponding to $a, b, c$ such that $\operatorname{discr}\left(a \mathcal{D}_{f} X+b \mathcal{D}_{f} Y+\right.$ $\left.c \mathcal{E}_{f}\right)=0$. Then $\operatorname{dim} W=2$. Take the component $W_{1}$ of $W$ which contains $L_{1}^{2} L_{2} \ldots L_{u}$. Then $W_{1}$ contains an open non-empty, hence two-dimensional, subset consisting of the operators $M_{1}^{2} M_{2} \ldots M_{u}$, where $M_{1}, \ldots, M_{u}$ are projectively different linear operators. If among $M_{2}, \ldots, M_{u}$ there are infinitely many projectively different linear operators, then it follows from Lemma 8.1 that for infinitely many linear forms $l_{u+1}, f+l_{u+1}^{d}$ is of minimal length $u+1$. Hence $f \prec g$ for infinitely many extreme forms $g$.

Otherwise, every operator from $W_{1}$ can be presented as $M^{2} L_{2} \ldots L_{u}$, where $M$ is linear and can be chosen to be projectively different from $L_{1}$. Then

$$
M^{2} L_{2} \ldots L_{u} f=M^{2} L_{2} \ldots L_{u} l_{1}^{d}=e l_{1}^{d-u-1}
$$

for some $e \in K^{*}$. This contradicts the assumption that $M^{2} L_{2} \ldots L_{u} \in$ $A_{u+1}(f)$.

This proves that there are infinitely many extreme forms $g$ such that $f \prec g$.

REMARK. It follows from the proof that, in fact, each $f$ as above is dominated by infinitely many extreme forms $g$ whose minimal length of presentation is maximal (equal to $d$ ). The number of remaining extreme forms dominating $f$ is finite.

Proof of Corollary 5. For $r(f) \leq(d+1) / 2$ the inequality $g \prec f$ has finitely many solutions by Lemma 5.2 of [1], and the reverse inequality has infinitely many solutions by Proposition 3. For $r(f)>(d+1) / 2$ the inequality $g \prec f$ has infinitely many solutions by Theorem 5 of [1] and the reverse inequality has finitely many solutions by Theorems 3 and 4 .

Proof of Proposition 4. Let $f=l_{1}^{d}+\cdots+l_{r}^{d}$ be a form and let $r=r(f) \leq$ $(d+1) / 2$. Then $\mathcal{D}_{f}=L_{1} \ldots L_{r}$, where $L_{i}$ are non-zero linear operators such that $L_{i} l_{i}=0$ for $i=1, \ldots, r$. We already know that always $\left(\mathcal{D}_{f}, \mathcal{E}_{f}\right)=1$. Let

$$
\mathcal{E}_{f}=M_{1}^{e_{1}+1} \ldots M_{u}^{e_{u}+1} M_{u+1} \ldots M_{u+t},
$$

where $M_{1}, \ldots, M_{u+t}$ are projectively distinct linear operators and

$$
0<e_{1}+\cdots+e_{u}+u<(d+2) / 2 .
$$

Let $m_{1}, \ldots, m_{u+t}$ be non-zero linear forms such that $M_{i} m_{i}=0, i=1, \ldots$, $u+t$. It follows from Lemma 2.1 that

$$
f=g_{1} m_{1}^{d-e_{1}}+\cdots+g_{u} m_{u}^{d-e_{u}}+a_{1} m_{u+1}^{d}+\cdots+a_{t} m_{u+t}^{d},
$$

where $g_{i}$ are forms of degree $e_{i}$ and $a_{j} \in K$, for $i=1, \ldots, u, j=1, \ldots, t$. Notice that $g_{i} \neq 0, a_{j} \neq 0$, since otherwise $f$ would be annihilated by a 
proper factor of $\mathcal{E}_{f}$. Then Theorem 2 implies that

$$
f_{0}=g_{1} m_{1}^{d-e_{1}}+\cdots+g_{u} m_{u}^{d-e_{u}}
$$

is extreme. Moreover, $f \preceq f_{0}$. Indeed,

$f_{0}=f-\left(a_{1} m_{u+1}^{d}+\cdots+a_{u+t} m_{u+t}^{d}\right)=l_{1}^{d}+\cdots+l_{r}^{d}-\left(a_{1} m_{u+1}^{d}+\cdots+a_{t} m_{u+t}^{d}\right)$

and this is a presentation of $f_{0}$ of minimal length, since

$$
\begin{aligned}
r\left(f_{0}\right) & =d-\left(e_{1}+\cdots+e_{u}+u\right)+2 \\
& =d-\left(e_{1}+\cdots+e_{u}+u+t\right)+t+2=d-\operatorname{deg} \mathcal{E}_{f}+t+2 \\
& =r(f)+t=r+t,
\end{aligned}
$$

and this is exactly the length of the above presentation of $f_{0}$. Thus $f \preceq f_{0}$. Also $r\left(f_{0}\right)>(d+1) / 2 \geq r(f)$. Hence $f \prec f_{0}$.

Conversely, let $f \prec f_{0}$, where $f_{0}$ is extreme. Then it follows from our assumptions and Theorem 2 that

$$
\begin{aligned}
f_{0} & =g_{1} m_{1}^{d-e_{1}}+\cdots+g_{u} m_{u}^{d-e_{u}}=l_{1}^{d}+\cdots+l_{t}^{d}, \\
f & =l_{1}^{d}+\cdots+l_{r}^{d}, \quad r<t,
\end{aligned}
$$

for some linear forms $m_{i}, l_{j}$ with $e_{1}+\cdots+e_{u}+u<(d+2) / 2$ and $t=$ $d-\left(e_{1}+\cdots+e_{u}+u\right)+2$. Thus

$$
f=g_{1} m_{1}^{d-e_{1}}+\cdots+g_{u} m_{u}^{d-e_{u}}-\left(l_{r+1}^{d}+\cdots+l_{t}^{d}\right)
$$

and an operator

$$
L_{r+1} \ldots L_{t} M_{1}^{e_{1}+1} \ldots M_{u}^{e_{u}+1}
$$

of degree

$t-r+e_{1}+\cdots+e_{u}+u=d-\left(e_{1}+\cdots+e_{u}+u\right)+2-r+e_{1}+\cdots+e_{u}+u=d-r+2$

annihilates $f$. This completes the proof.

\section{Proof of Theorem 5}

Lemma 9.1. If $A(f)=A(g)$, then $f / g \in K \backslash\{0\}$.

Proof. See [2, Lemma 2.12].

Lemma 9.2. A regular form of degree $2 m$ is extreme if and only if either it is equivalent via a linear transformation to $x^{m} y^{m}$, or in $A_{m+1}(f)$ there are exactly three projectively distinct non-zero forms with a square factor and each of them is squareful.

Proof. By Lemma 2.2, $A_{m+1}(f)$ is generated by two coprime forms $\mathcal{D}_{1}$ and $\mathcal{D}_{2}$ of degree $m+1$. Let

$$
B_{1}(X)=\mathcal{D}_{1}(X, 1), \quad B_{2}(X)=\mathcal{D}_{2}(X, 1) .
$$


Since $\mathcal{D}_{1}, \mathcal{D}_{2}$ are not both divisible by $Y$ we shall assume without loss of generality that $\operatorname{deg} B_{1}=m+1$ and that $B_{1}, B_{2}$ are monic. Let

$$
C= \begin{cases}B_{2} & \text { if } \operatorname{deg} B_{2}<\operatorname{deg} B_{1} \\ B_{2}-B_{1} & \text { if } \operatorname{deg} B_{2}=\operatorname{deg} B_{1}\end{cases}
$$

so that $n=\operatorname{deg} C \leq m$, and let the leading coefficient of $C$ be $c$. It follows from

$$
B_{1}^{\prime} B_{2}-B_{1} B_{2}^{\prime}=B_{1}^{\prime} C-B_{1} C^{\prime}
$$

(prime denotes differentiation) that the coefficient of $X^{m+n}$ in $B_{1}^{\prime} B_{2}-B_{1} B_{2}^{\prime}$ is $c(m+1-n) \neq 0$, hence $B_{1}^{\prime} B_{2}-B_{1} B_{2}^{\prime}$ is of degree $m+n>0$, thus the set $Z$ of its zeros is not empty. Let

$$
B_{1}^{\prime} B_{2}-B_{1} B_{2}^{\prime}=c(m+1-n) \prod_{z \in Z}(X-z)^{e(z)} .
$$

It follows by induction on $i$ that for every $z \in Z$ and for every positive integer $i \leq e(z)$,

$$
B_{1}^{(i)}(z) B_{2}(z)-B_{1}(z) B_{2}^{(i)}(z)=0 .
$$

For every $z_{0} \in Z$ the form

$$
F=B_{2}\left(z_{0}\right) \mathcal{D}_{1}-B_{1}\left(z_{0}\right) \mathcal{D}_{2} \in A_{m+1}(f)
$$

has the square factor $\left(X-z_{0} Y\right)^{2}$. Moreover $F \neq 0$ since $\left(\mathcal{D}_{1}, \mathcal{D}_{2}\right)=1$ and $B_{j}\left(z_{0}\right) \neq 0$ for some $j=1,2$. If $F$ is, up to projective equality, the only non-zero element of $A_{m+1}(f)$ with a square factor, then for every $z \in Z$ we have $B_{j}(z) \neq 0$ and

$$
\frac{B_{3-j}(z)}{B_{j}(z)}=\frac{B_{3-j}\left(z_{0}\right)}{B_{j}\left(z_{0}\right)} .
$$

Now, by (22),

$$
P=B_{3-j}(X)-\frac{B_{3-j}\left(z_{0}\right)}{B_{j}\left(z_{0}\right)} B_{j}(X)
$$

contains the factor $X-z$ with multiplicity $e(z)+1$, hence by (21),

$$
m+1 \geq \operatorname{deg} P \geq \operatorname{deg}\left(B_{1}^{\prime} B_{2}-B_{1} B_{2}^{\prime}\right)+|Z|=m+n+|Z|>m+n,
$$

thus $n=0$. It follows that $B_{2}-B_{1}=b \in K \backslash\{0\}$, hence

$$
b Y^{m+1}=\mathcal{D}_{2}-\mathcal{D}_{1} \in A_{m+1}(f)
$$

and $A_{m+1}(f)$ contains at least two non-zero, projectively distinct elements with a square factor, namely $F$ and $Y^{m+1}$. This contradiction proves that the number of projectively distinct non-zero elements of $A_{m+1}(f)$ with a square factor is at least two.

We proceed to prove that there are at most three such elements. Let $F_{1}, F_{2}$ be two of them and suppose that

$$
a_{1} F_{1}+a_{2} F_{2}=F_{3}, \quad b_{1} F_{1}+b_{2} F_{2}=F_{4},
$$


where $a_{i}, b_{i} \in K \backslash\{0\}, F_{3}, F_{4}$ have a square factor and are projectively distinct. Since $\left(\mathcal{D}_{1}, \mathcal{D}_{2}\right)=1$ the forms $F_{i}(1 \leq i \leq 4)$ are coprime, i.e. pairwise relatively prime, and since $f$ is extreme, $F_{i}$ are squareful. Let

$$
\bar{F}_{i}(X)=F_{i}(X, 1)=\prod_{z \in Z_{i}}(X-z)^{e_{i}(z)} .
$$

We see that $Z_{i}$ are disjoint (one $Z_{i}$ may be empty) and

$$
e_{i}(z) \geq 2 \quad\left(1 \leq i \leq 4, z \in Z_{i}\right) .
$$

From (20) we obtain

$$
\begin{aligned}
a_{1} \bar{F}_{1}+a_{2} \bar{F}_{2} & =\bar{F}_{3}, \\
b_{1} \bar{F}_{1}+b_{2} \bar{F}_{2} & =\bar{F}_{4},
\end{aligned}
$$

and by differentiation

$$
\begin{aligned}
& a_{1}\left(\bar{F}_{1}^{\prime} \bar{F}_{2}-\bar{F}_{1} \bar{F}_{2}^{\prime}\right)=\bar{F}_{3}^{\prime} \bar{F}_{2}-\bar{F}_{3} \bar{F}_{2}^{\prime}, \\
& b_{1}\left(\bar{F}_{1}^{\prime} \bar{F}_{2}-\bar{F}_{1} \bar{F}_{2}^{\prime}\right)=\bar{F}_{4}^{\prime} \bar{F}_{2}-\bar{F}_{4} \bar{F}_{2}^{\prime},
\end{aligned}
$$

thus

$$
\prod_{i=1}^{4}\left(\bar{F}_{i}^{\prime}, \bar{F}_{i}\right) \mid\left(\bar{F}_{1}^{\prime} \bar{F}_{2}-\bar{F}_{1} \bar{F}_{2}^{\prime}\right) .
$$

By (24) and (25),

$$
\operatorname{deg}\left(\bar{F}_{i}^{\prime}, \bar{F}_{i}\right) \geq \frac{1}{2} \operatorname{deg} \bar{F}_{i} .
$$

If $\operatorname{deg} \bar{F}_{1} \neq \operatorname{deg} \bar{F}_{2}$, then

$$
\operatorname{deg} \bar{F}_{3}=\operatorname{deg} \bar{F}_{4}=\max \left(\operatorname{deg} \bar{F}_{1}, \operatorname{deg} \bar{F}_{2}\right)
$$

and (26) gives

$$
\begin{aligned}
& \frac{3}{2} \max \left(\operatorname{deg} \bar{F}_{1}, \operatorname{deg} \bar{F}_{2}\right)+\frac{1}{2} \min \left(\operatorname{deg} \bar{F}_{1}, \operatorname{deg} \bar{F}_{2}\right) \\
& \leq \max \left(\operatorname{deg} \bar{F}_{1}, \operatorname{deg} \bar{F}_{2}\right)+\min \left(\operatorname{deg} \bar{F}_{1}, \operatorname{deg} \bar{F}_{2}\right)-1,
\end{aligned}
$$

a contradiction. If $\operatorname{deg} \bar{F}_{i}$ are equal, then the same argument applies. If $\operatorname{deg} \bar{F}_{1}=\operatorname{deg} \bar{F}_{2}$ but $\operatorname{deg} \bar{F}_{j}<\operatorname{deg} \bar{F}_{1}$ for $j=3$ or 4 , say $j=3$, we have

$$
\bar{F}_{1}^{\prime} \bar{F}_{2}-\bar{F}_{1} \bar{F}_{2}^{\prime}=\frac{1}{a_{2}}\left(\bar{F}_{1}^{\prime} \bar{F}_{3}-\bar{F}_{1} \bar{F}_{3}^{\prime}\right)
$$

and (27) holds with $\bar{F}_{2}$ replaced by $\bar{F}_{3}$. This contradiction shows that the number of projectively distinct non-zero elements of $A_{m+1}(f)$ with a square factor is at most three.

It remains to prove that for a form $f$ in question, $A_{m+1}(f)$ contains exactly two projectively distinct non-zero forms with a square factor if and 
only if $f$ is equivalent to $x^{m} y^{m}$. Let the two relevant forms in $A_{m+1}(f)$ be $F_{1}$ and $F_{2}$, and let $\bar{F}_{i}(X)=F_{i}(X, 1)$ for $i=1,2$. We have

$$
\left(\bar{F}_{1}, \bar{F}_{2}\right)=1
$$

and we assert that

$$
\bar{F}_{1}^{\prime} \bar{F}_{2}-\bar{F}_{1} \bar{F}_{2}^{\prime}=c_{1}\left(\bar{F}_{1}, \bar{F}_{1}^{\prime}\right)\left(\bar{F}_{2}, \bar{F}_{2}^{\prime}\right), \quad c_{1} \in K \backslash\{0\} .
$$

If it were not so, then

$$
H=\frac{\bar{F}_{1}^{\prime} \bar{F}_{2}-\bar{F}_{1} \bar{F}_{2}^{\prime}}{\left(\bar{F}_{1}, \bar{F}_{1}^{\prime}\right)\left(\bar{F}_{2}, \bar{F}_{2}^{\prime}\right)}
$$

would have a zero $\zeta$. If $\zeta$ were a zero of $\bar{F}_{i}$ with multiplicity $m_{1}>0$, then $\zeta$ would be a zero of $\bar{F}_{i} /\left(\bar{F}_{i}, \bar{F}_{i}^{\prime}\right)$ of multiplicity 1 , hence $H(\zeta)=0$ would imply

$$
\frac{\bar{F}_{i}^{\prime} \bar{F}_{3-i}}{\left(\bar{F}_{1}, \bar{F}_{1}^{\prime}\right)\left(\bar{F}_{2}, \bar{F}_{2}^{\prime}\right)}(\zeta)=0
$$

and since $\frac{\bar{F}_{i}^{\prime}}{\left(\bar{F}_{i}, \bar{F}_{i}^{\prime}\right)}(\zeta) \neq 0$ we should obtain $\bar{F}_{3-i}(\zeta)=0$, contrary to (28). Therefore $\bar{F}_{1}(\zeta) \bar{F}_{2}(\zeta) \neq 0$ and the form $\bar{F}_{2}(\zeta) F_{1}-\bar{F}_{1}(\zeta) F_{2}$ projectively distinct from $F_{1}, F_{2}$ would have the square factor $(X-\zeta Y)^{2}$. This proves (29).

Now, suppose that $\bar{F}_{i}$ has exactly $q_{i}$ projectively distinct factors and $q_{1} \geq q_{2}$. Then

$$
\operatorname{deg}\left(\bar{F}_{1}, \bar{F}_{1}^{\prime}\right)\left(\bar{F}_{2}, \bar{F}_{2}^{\prime}\right)=\operatorname{deg} \bar{F}_{1}+\operatorname{deg} \bar{F}_{2}-q_{1}-q_{2}
$$

and

$$
\operatorname{deg}\left(\bar{F}_{1}^{\prime} \bar{F}_{2}-\bar{F}_{1} \bar{F}_{2}^{\prime}\right)=\operatorname{deg} \bar{F}_{1}+\operatorname{deg} \bar{F}_{2}-1 \quad \text { if } \operatorname{deg} \bar{F}_{1} \neq \operatorname{deg} \bar{F}_{2} .
$$

This gives, by (29), that $q_{1}=1, q_{2}=0$, thus $\bar{F}_{1}$ is a power of a linear polynomial, $\bar{F}_{2} \in K \backslash\{0\}$. It follows that $F_{1}=L^{m+1}, F_{2}=Y^{m+1}$, where $L$ is a linear form and by a linear transformation we can achieve that $F_{1}=X^{m+1}$, $F_{2}=Y^{m+1}$. Then $f$ annihilated by $F_{1}$ and $F_{2}$ is projectively equal to $x^{m} y^{m}$.

It remains to consider the case where $\operatorname{deg} \bar{F}_{1}=\operatorname{deg} \bar{F}_{2}$. Then $\bar{F}_{2}=$ $c_{3} \bar{F}_{1}+R$, where $c_{3} \neq 0, \operatorname{deg} R<\operatorname{deg} \bar{F}_{1}$. If $\operatorname{deg} R \leq \operatorname{deg} \bar{F}_{1}-2$, then $F_{2}-c_{3} F_{1}$ is divisible by $Y^{2}$ and projectively distinct from $F_{1}, F_{2}$, contrary to the assumption. Thus $\operatorname{deg} R=\operatorname{deg} \bar{F}_{1}-1$ and $\operatorname{deg}\left(\bar{F}_{1}^{\prime} \bar{F}_{2}-\bar{F}_{1} \bar{F}_{2}^{\prime}\right)=\operatorname{deg}\left(\bar{F}_{1}^{\prime} R-\bar{F}_{1} R^{\prime}\right)=\operatorname{deg} \bar{F}_{1}+\operatorname{deg} R-1=2 \operatorname{deg} \bar{F}_{1}-2$. Hence from (29) and (30) we obtain

$$
2 \operatorname{deg} \bar{F}_{1}-2=2 \operatorname{deg} \bar{F}_{1}-q_{1}-q_{2},
$$

thus $q_{1}+q_{2}=2, q_{1}=q_{2}=1, \bar{F}_{i}=L_{i}(X, 1)^{m+1}$, where $L_{i}$ is a linear form, $F_{i}=L_{i}^{m+1}$ and the previous argument shows that $f$ annihilated via $F_{1}, F_{2}$ is equivalent via a linear transformation to $x^{m} y^{m}$. 
Conversely, if $f=x^{m} y^{m}$, then $A_{m+1}(f)$ contains $X^{m+1}, Y^{m+1}$ and up to a constant factor no other form with a square factor.

Lemma 9.3. For every positive integer $s$ there exist up to a fractional linear transformation only finitely many rational functions $a, b \in K(x) \backslash\{0\}$ with the set $S$ of zeros and poles such that $a+b=1,|S|=s$ and $-\sum_{p \in S} \min \left\{\operatorname{ord}_{p} a, \operatorname{ord}_{p} b\right\}=s-2$.

Proof. See [6, Theorem 3] in which one takes $k \mapsto K, K \mapsto K(x)$.

Proof of Theorem 5. We begin by proving that the condition is necessary. By Lemma 9.2 it suffices to show that if $P, Q, R$ are three projectively distinct forms in $A_{m+1}(f)$, then they are coprime and the total number of their projectively distinct linear factors is $m+3$. The former fact results from Lemma 2.3. Also, replacing if necessary $P, Q, R$ by their scalar multiples, we may assume that $R=P+Q$.

By a linear transformation we can achieve that $Y \mid Q$. Now, let

$$
\bar{P}=P(X, 1), \quad \bar{Q}=Q(X, 1), \quad \bar{R}=R(X, 1)
$$

and let $\omega(\bar{P}), \omega(\bar{Q}), \omega(\bar{R})$ denote the number of zeros of $\bar{P}, \bar{Q}, \bar{R}$, respectively. Since $P$ and $Q$ cannot be both divisible by $Y$ we have

$$
\operatorname{deg} \bar{P}=\operatorname{deg} \bar{R}=m+1>\operatorname{deg} \bar{Q},
$$

and the $a b c$-theorem (see [6]) gives

$$
\omega(\bar{P})+\omega(\bar{Q})+\omega(\bar{R}) \geq m+2 .
$$

We shall show that equality holds here. Therefore assume

$$
\omega(\bar{P})+\omega(\bar{Q})+\omega(\bar{R}) \geq m+3 .
$$

Since

$$
\begin{aligned}
& \operatorname{deg}\left(\bar{P}, \bar{P}^{\prime}\right)=\operatorname{deg} \bar{P}-\omega(\bar{P}), \quad \operatorname{deg}\left(\bar{Q}, \bar{Q}^{\prime}\right)=\operatorname{deg} \bar{Q}-\omega(\bar{Q}), \\
& \operatorname{deg}\left(\bar{R}, \bar{R}^{\prime}\right)=\operatorname{deg} \bar{R}-\omega(\bar{R}),
\end{aligned}
$$

inequalities (31) and (32) give

$$
\operatorname{deg}\left(\bar{P}^{\prime} \bar{Q}-\bar{P} \bar{Q}^{\prime}\right)=\operatorname{deg} \bar{Q}+m>\operatorname{deg}\left(\bar{P}, \bar{P}^{\prime}\right)+\operatorname{deg}\left(\bar{Q}, \bar{Q}^{\prime}\right)+\operatorname{deg}\left(\bar{R}, \bar{R}^{\prime}\right) .
$$

The polynomials $\left(\bar{P}, \bar{P}^{\prime}\right),\left(\bar{Q}, \bar{Q}^{\prime}\right),\left(\bar{R}, \bar{R}^{\prime}\right)$ are coprime and each of them divides $\bar{P}^{\prime} \bar{Q}-\bar{P} \bar{Q}^{\prime}$. For the first two this is evident, for the third it follows from the identity

$$
2\left(\bar{P}^{\prime} \bar{Q}-\bar{P} \bar{Q}^{\prime}\right)=(\bar{P}+\bar{Q})\left(\bar{P}^{\prime}-\bar{Q}^{\prime}\right)-\left(\bar{P}^{\prime}+\bar{Q}^{\prime}\right)(\bar{P}-\bar{Q}) .
$$

By virtue of (33),

$$
\frac{\bar{P}^{\prime} \bar{Q}-\bar{P} \bar{Q}^{\prime}}{\left(\bar{P}, \bar{P}^{\prime}\right)\left(\bar{Q}, \bar{Q}^{\prime}\right)\left(\bar{R}, \bar{R}^{\prime}\right)}
$$


has a zero $\zeta$. If we had $\bar{P}(\zeta)=0$ it would follow that

$$
\frac{\bar{P}^{\prime} \bar{Q}}{\left(\bar{P}, \bar{P}^{\prime}\right)}(\zeta)=0,
$$

contrary to

$$
\left(\bar{P}, \frac{\bar{P}^{\prime} \bar{Q}}{\left(\bar{P}, \bar{P}^{\prime}\right)}\right)=1 .
$$

This contradiction shows that $\bar{P}(\zeta) \neq 0$ and similarly $\bar{Q}(\zeta) \neq 0$, while identity (34) implies $\bar{R}(\zeta) \neq 0$. Now consider the form

$$
F=\bar{Q}(\zeta) P-\bar{P}(\zeta) Q
$$

$F$ has the square factor $(X-\zeta Y)^{2}$, since $\bar{Q}(\zeta) \bar{P}-\bar{P}(\zeta) \bar{Q}$ has $\zeta$ as a multiple zero. However, since $(P Q R)(\zeta) \neq 0, F$ is projectively different from $P, Q, R$ contrary to Lemma 9.2. This contradiction shows that

$$
\omega(\bar{P})+\omega(\bar{Q})+\omega(\bar{R})=m+2 .
$$

Since $Q$ is divisible by $Y$, the number of projectively distinct linear factors of $P Q R$ is $m+3$.

We proceed to prove that the condition is sufficient. If $P, Q, R$ are three coprime squareful forms in $A_{m+1}(f)$ with the number of projectively distinct linear factors of $P Q R$ equal to $m+3$ we may assume that $P+Q=R$ and $Q$ is divisible by $Y$. Let again

$$
\bar{P}=P(X, 1), \quad \bar{Q}=Q(X, 1), \quad \bar{R}=R(X, 1) .
$$

The condition on $P Q R$ now gives

$$
\omega(\bar{P})+\omega(\bar{Q})+\omega(\bar{R})=m+2 .
$$

By Lemma 9.2, it suffices to show that every non-zero form $F=a P+b Q$ $(a, b \in K)$ with a square factor is projectively equal $(\simeq)$ to $P, Q$ or $R$. If $Y^{2} \mid F$, then since $Y \mid Q$ and $Y \nmid P$, we have $a=0$ and $F \simeq Q$. If $F$ has a square factor and $Y^{2} \nmid F$, then $a \bar{P}+b \bar{Q}$ has a multiple zero $\zeta$. Thus

$$
a \bar{P}(\zeta)+b \bar{Q}(\zeta)=0, \quad a \bar{P}^{\prime}(\zeta)+b \bar{Q}^{\prime}(\zeta)=0,
$$

hence

$$
\left(\bar{P}^{\prime} \bar{Q}-\bar{P} \bar{Q}^{\prime}\right)(\zeta)=0 .
$$

However, as shown in the first part of the proof,

$$
\left(\bar{P}, \overline{P^{\prime}}\right)\left(\bar{Q}, \bar{Q}^{\prime}\right)\left(\bar{R}, \bar{R}^{\prime}\right) \mid\left(\bar{P}^{\prime} \bar{Q}-\bar{P} \bar{Q}^{\prime}\right) .
$$

Now, by (35),

$$
\begin{aligned}
\operatorname{deg}\left(\bar{P}, \bar{P}^{\prime}\right)\left(\bar{Q}, \bar{Q}^{\prime}\right)\left(\bar{R}, \bar{R}^{\prime}\right) & =\operatorname{deg} \bar{P}+\operatorname{deg} \bar{Q}+\operatorname{deg} \bar{R}-\omega(\bar{P})+\omega(\bar{Q})-\omega(\bar{R}) \\
& =m+\operatorname{deg} \bar{Q}=\operatorname{deg}\left(\bar{P}^{\prime} \bar{Q}-\bar{P} \bar{Q}^{\prime}\right)
\end{aligned}
$$


hence

$$
\bar{P}^{\prime} \bar{Q}-\bar{P} \bar{Q}^{\prime}=c\left(\bar{P}, \bar{P}^{\prime}\right)\left(\bar{Q}, \bar{Q}^{\prime}\right)\left(\bar{R}, \bar{R}^{\prime}\right), \quad c \in K \backslash\{0\} .
$$

Therefore (37) implies $\bar{P}(\zeta)=0$ or $\bar{Q}(\zeta)=0$ or $\bar{R}(\zeta)=0$. If $\bar{P}(\zeta)=0$, then $\bar{Q}(\zeta) \neq 0$ and (36) gives $b=0$, thus $F \simeq P$. If $\bar{Q}(\zeta)=0$, then similarly $a=0$, thus $F \simeq Q$. Finally, if $\bar{R}(\zeta)=0$, then $\bar{P}(\zeta)+\bar{Q}(\zeta)=0$, thus by (36), $a=b$ and $F \simeq R$.

Proof of Corollary 6 . We begin with a remark valid for every $m$. If any of the three squareful forms is a power of a linear form, then, since each of the others has at most $(m+1) / 2$ projectively distinct linear factors, the total number of such factors is too small, namely at most $m+2$. Thus none of the three forms is a power of a linear form, which settles the case $m \leq 2$, where every squareful form is of this type. For $m=3$ the relevant squareful forms are of the type $X^{2} Y^{2}$, thus we have $P=P_{1}^{2}, Q=Q_{1}^{2}, R=R_{1}^{2}$ where $P_{1}, Q_{1}, R_{1}$ have one linear factor each and $P_{1}^{2}+Q_{1}^{2}=R_{1}^{2}$. Therefore $R_{1}+P_{1}$, $R_{1}-P_{1}$ have one linear factor each, and taking them equal to $2 X^{2}, 2 Y^{2}$ we obtain

$$
P=\left(X^{2}-Y^{2}\right)^{2}, \quad Q=4 X^{2} Y^{2}, \quad R=\left(X^{2}+Y^{2}\right)^{2}, \quad f \simeq x^{5} y-x y^{5} .
$$

For $m=4$ the relevant squareful forms are of type $X^{3} Y^{2}$, thus the total number of projectively distinct linear factors of three such forms is $6<m+3$. For $m=5$ the relevant squareful forms are of type $X^{4} Y^{2}, X^{3} Y^{3}$, or $X^{2} Y^{2} Z^{2}$. The condition on the total number of projectively distinct linear factors leads to $P=P_{1}^{2}, R=R_{1}^{2}$, where $P_{1}, R_{1}$ have three factors each, and $Q=R-P$ has two such factors. Therefore, $R_{1}+P_{1}, R_{1}-P_{1}$ have one linear factor each, and taking them equal to $2 X^{3}, 2 Y^{3}$ we obtain

$$
P=\left(X^{3}-Y^{3}\right)^{2}, \quad Q=X^{3} Y^{3}, \quad R=\left(X^{3}+Y^{3}\right)^{2}, \quad f \simeq x^{8} y^{2}-x^{2} y^{8} .
$$

Proof of Corollary 7 . For a form $f$ of degree $2 m$, coprime polynomials $P, Q \in A_{m+1}(f)$ determine $A(f)$ by virtue of Lemma 2.2, hence by Lemma 9.1, $f$ is determined by them up to a constant factor. Transforming $P, Q$ by a linear substitution results in transforming $f$ by the inverse of that substitution, thus it suffices to prove that up to a linear substitution there are only finitely many triples $P, Q, R=P+Q$ of coprime forms of degree $m+1$, which together have exactly $m+3$ projectively distinct linear factors. Assuming without loss of generality that $Y \mid Q$ and putting

$$
\bar{P}=P(X, 1), \quad \bar{Q}=Q(X, 1), \quad \bar{R}=R(X, 1)
$$

we obtain

$$
\omega(\bar{P})+\omega(\bar{Q})+\omega(\bar{R})=m+2 .
$$


Now, we apply Lemma 9.3 with $a=\bar{P} / \bar{R}$ and $b=\bar{Q} / \bar{R}$. Here

$$
\begin{gathered}
-\sum_{p \in S} \min \left\{\operatorname{ord}_{p} a, \operatorname{ord}_{p} b\right\}=\operatorname{deg} \bar{R}=m+1, \\
|S|=\omega(\bar{P})+\omega(\bar{Q})+\omega(\bar{R})=m+3 .
\end{gathered}
$$

Thus the assumptions of the lemma are satisfied and so there exist, up to a fractional linear transformation, only finitely many pairs $\bar{P} / \bar{R}, \bar{Q} / \bar{R}$ in question. It follows that up to a linear transformation there exist only finitely many relevant triples $P, Q, R$.

Example (due to J. Browkin). For $m=6$ the conditions of Theorem 5 are satisfied by the following forms in $K[X, Y]_{m+1}$ :

$$
\begin{aligned}
& P=(X-Y)^{3}\left(4 X^{2}-X Y+Y^{2}\right)^{2}, \quad Q=Y^{3}\left(7 X^{2}-7 X Y+4 Y^{2}\right)^{2}, \\
& R=X^{3}\left(7 X Y+7 Y^{2}\right)^{2} .
\end{aligned}
$$

10. Proof of Proposition 5. In all three cases we argue in the same way. First we notice that, in all three cases, the number on the right hand side is equal to $d-s(f)+2=\operatorname{deg} \mathcal{E}_{f}$. Denote that number by $t(f)$. Next notice that $\operatorname{dim} A_{t(f)}(f) \geq 2, t(f) \leq \bar{r}(f)$. Moreover, $A_{t(f)}(f)$ contains coprime forms and contains a Zariski dense subset consisting of squarefree forms. Then it follows from Lemma 2.1 that it is sufficient to prove that, for any given finite family $M_{1}, \ldots, M_{t}$ of projectively distinct linear operators, we can find linear forms $L_{1}, \ldots, L_{r}$ such that all forms $L_{1}, \ldots, L_{r}, M_{1}, \ldots, M_{t}$ are pairwise projectively distinct and $G=L_{1} \ldots L_{r} \in A_{t(f)}(f)$. However, for $i=1, \ldots, r, M_{i}$ does not divide all forms from $A_{t(f)}(f)$, since $A_{t(f)}$ contains coprime forms. Thus forms in $A_{t(f)}(f)$ divisible by $M_{i}$ belong to a hyperplane and hence for a Zariski dense subset $U \subset A_{t(f)}(f)$ the condition $G \in U$ implies that $M_{i} \nmid G$ for all $i=1, \ldots, r$. On the other hand, as noticed above, for a Zariski dense subset $V \subset A_{t(f)}(f)$ the condition $G \in V$ implies that $G$ is squarefree. Hence, $U \cap V \neq \emptyset$ and every form $G \in U \cap V$ satisfies the required conditions.

Acknowledgments. The research of A. Białynicki-Birula was partially supported by Polish MNiSW Grant N201 019 32/0805.

\section{References}

[1] A. Białynicki-Birula and A. Schinzel, Representations of multivariate polynomials as sums of univariate polynomials in linear forms, Colloq. Math. 112 (2008), 201-233.

[2] A. Iarrobino and V. Kanev, Power Sums, Gorenstein Algebras and Determinantal Loci, Lecture Notes in Math. 1721, Springer, 1999. 
[3] J. Kleppe, Representing a homogeneous polynomial as a sum of powers of linear forms, Thesis for the degree of Candidatus Scientiarum, Department of Mathematics, Univ. of Oslo, 1999.

[4] Th. Muir, A Treatise on the Theory of Determinants, Dover, 1960.

[5] J. J. Sylvester, On a remarkable discovery in the theory of canonical forms and of hyperdeterminant, in: The Collected Mathematical Papers, Cambridge, 1904, 215263.

[6] U. Zannier, Some remarks on the S-unit equation in function fields, Acta Arith. 64 (1993), 87-98.

Andrzej Białynicki-Birula

Department of Mathematics

University of Warsaw

Banacha 2

02-097 Warszawa, Poland

E-mail: bbirula@mimuw.edu.pl
Andrzej Schinzel

Institute of Mathematics

Polish Academy of Sciences

P.O. Box 21

00-956 Warszawa, Poland

E-mail: schinzel@impan.gov.pl

Received on 15.5.2009

and in revised form on 5.10.2009 